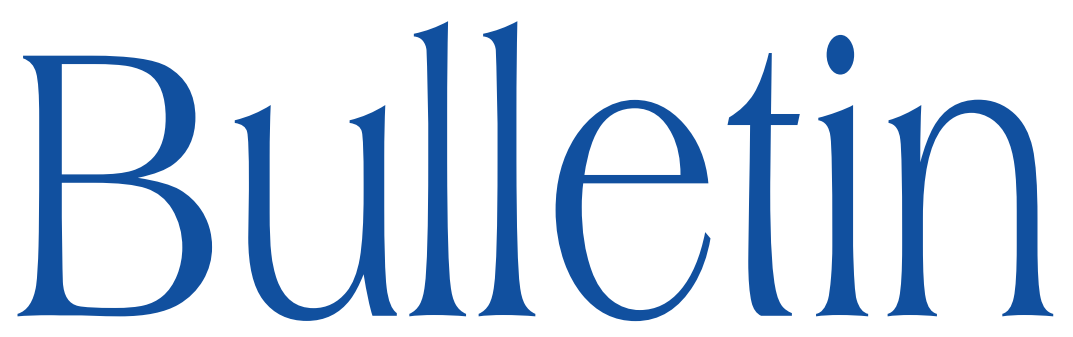

de la SOCIÉTÉ MATHÉMATIQUE DE FRANCE

\title{
PSEUDO-SPECTRUM FOR A CLASS OF SEMI-CLASSICAL OPERATORS
}

Karel Pravda-Starov

Tome 136

Fascicule 3

2008 
Bull. Soc. math. France

136 (3), 2008, p. 329-372

\title{
PSEUDO-SPECTRUM FOR A CLASS OF SEMI-CLASSICAL OPERATORS
}

\author{
BY KAREL PRAVDA-StaROV
}

\begin{abstract}
We study in this paper a notion of pseudo-spectrum in the semi-classical setting called injectivity pseudo-spectrum. The injectivity pseudo-spectrum is a subset of points in the complex plane where there exist some quasi-modes with a precise rate of decay. For that reason, these values can be considered as some 'almost eigenvalues' in the semi-classical limit. We are interested here in studying the absence of injectivity pseudo-spectrum, which is characterized by a global a priori estimate. We prove in this paper a sharp global subelliptic a priori estimate for a class of pseudo-differential operators with respect to the regularity of their symbols. Our main result extends the a priori estimate of Dencker, Sjöstrand and Zworski for a class of pseudo-differential operators with symbols of limited smoothness violating the condition $(P)$.

RÉSumÉ (Pseudo-spectre d'une classe d'opérateurs semi-classiques)

Nous étudions dans cet article une notion de pseudo-spectre semi-classique appelée pseudo-spectre d'injectivité. Le pseudo-spectre d'injectivité d'un opérateur désigne l'ensemble des points du plan complexe qui sont des «presque valeurs propres » dans l'asymptotique semi-classique, au sens où il existe en ces points des quasi-modes semiclassiques avec des taux précis de décroissance. Nous nous intéressons ici à l'étude de l'absence de pseudo-spectre d'injectivité, et nous démontrons une estimation souselliptique globale pour une classe d'opérateurs pseudo-différentiels dont les symboles ont une régularité limitée. Ce résultat généralise dans un cadre de régularité limitée l'estimation a priori démontrée par Dencker, Sjöstrand et Zworski pour une classe d'opérateurs pseudo-différentiels violant la condition $(P)$.
\end{abstract}

Texte reçu le 4 octobre 2006, révisé le 16 juillet 2007

Karel Pravda-Starov, Department of Mathematics, University of California, Los Angeles, CA 90095-1555, USA • E-mail : karel@math.ucla.edu

2000 Mathematics Subject Classification. - 35S05.

Key words and phrases. - Subelliptic estimate, symbols with limited smoothness, condition $(\bar{\Psi})$, Wick quantization. 


\section{Introduction}

1.1. Miscellaneous facts about pseudo-spectrum. - In recent years, there has been a lot of interest in studying the pseudo-spectrum of non-self-adjoint operators. We first recall some classical and known facts about pseudo-spectrum. The study of pseudo-spectrum has been initiated by noticing that for certain problems of science and engineering involving non-self-adjoint operators, the predictions suggested by spectral analysis do not match with the numerical simulations. To supplement the lack of information given by the spectrum, some new subsets of the complex plane called pseudo-spectra have been defined. The main idea is to study not only points where the resolvent is not defined, i.e., the spectrum, but also where the resolvent is large in norm.

We refer the reader to Trefethen's article [11] ${ }^{(1)}$ for the definition of the $\varepsilon$-pseudo-spectrum $\Lambda_{\varepsilon}(A)$ of a matrix or an operator $A$,

$$
\Lambda_{\varepsilon}(A)=\left\{z \in \mathbb{C},\left\|(z I-A)^{-1}\right\| \geq \varepsilon^{-1}\right\} .
$$

By convention, we write $\left\|(z I-A)^{-1}\right\|=+\infty$ if $z$ belongs to the spectrum of $A$. The $\varepsilon$-pseudo-spectrum of $A$ is non-decreasing with $\varepsilon$. All these subsets contain the spectrum of the operator. In an equivalent way, pseudo-spectra can be defined in term of the spectra of perturbations. Indeed, for any matrix we have

$$
\Lambda_{\varepsilon}(A)=\{z \in \mathbb{C}, z \in \sigma(A+B) \text { for some } B \text { with }\|B\| \leq \varepsilon\} .
$$

It follows that a number $z$ belongs to the $\varepsilon$-pseudo-spectrum of $A$ if and only if it belongs to the spectrum of some perturbed operator $A+B$ with $\|B\| \leq$ $\varepsilon$. From this second description, we understand the interest in studying such subsets. Indeed, if we want to compute numerically some eigenvalues, we start by discretizing the operator. This discretization and inevitable round-off errors will generate some perturbations of the initial operator. Eventually, algorithms for eigenvalues computing determine the eigenvalues of a perturbation of the initial operator, i.e., a value in some $\varepsilon$-pseudo-spectrum and not necessarily a spectral value. In the self-adjoint case, the spectrum is stable under small perturbations. In fact, this stability is a consequence of the spectral theorem. The spectral theorem implies that $\Lambda_{\varepsilon}(A)$ is exactly the set of points in $\mathbb{C}$ at distance less than or equal to $\varepsilon$ from the spectrum of $A$. However this property of stability is not true in the non-self-adjoint case in which the spectrum could be very unstable under small perturbations. To illustrate this fact, we recall a suggestive example pointed out by Davies in [3] and Zworski in [12].

(1) The reader will find in this paper more details about interest, history and general properties of pseudo-spectra.

TOME $136-2008-\mathrm{N}^{\mathrm{O}} 3$ 
EXAMPLE. - Let us consider the rotated harmonic oscillator in one dimension $P_{\alpha}=-\frac{d^{2}}{d x^{2}}+e^{i \alpha} x^{2}$ where $-\pi<\alpha<\pi$.
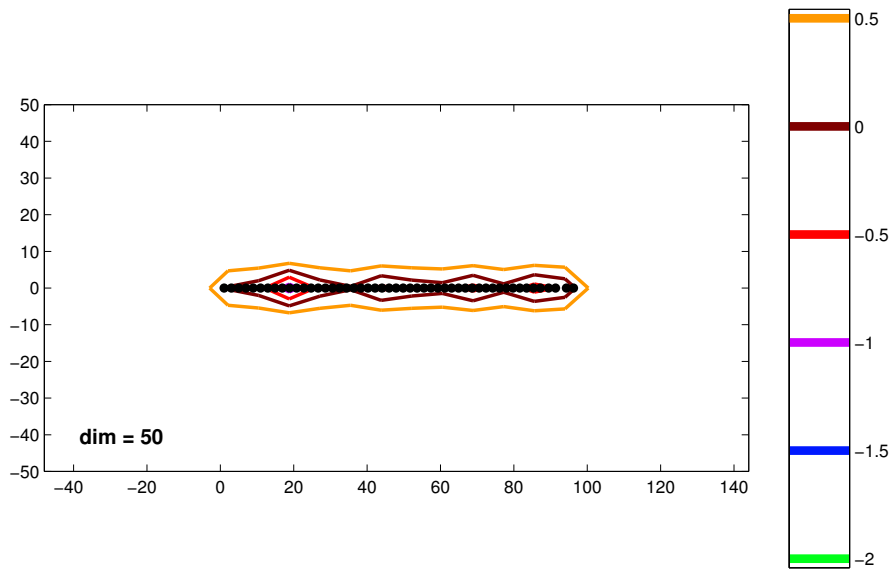

Figure 1. Computation of $\varepsilon$-pseudo-spectra for the rotated harmonic oscillator $P_{\alpha}$ with $\alpha=0$. The right column gives the corresponding values of $\log _{10} \varepsilon$.

This operator $P_{\alpha}$ is self-adjoint only for $\alpha=0$. Its spectrum is composed of the following eigenvalues (see Theorem 3.3 in [6]),

$$
e^{i \frac{\alpha}{2}}(2 n+1), n \in \mathbb{N} \text {. }
$$

We can try to compute numerically the spectrum and some $\varepsilon$-pseudo-spectra for some small values of the parameter $\varepsilon$. Computations are performed on the discretization

$$
\left(\left(P_{\alpha} \Psi_{i}, \Psi_{j}\right)_{L^{2}(\mathbb{R})}\right)_{1 \leq i, j \leq N},
$$

where $N$ is an integer taken equal to 50 and $\left(\Psi_{j}\right)_{j \in \mathbb{N}^{*}}$ stands for the basis of $L^{2}(\mathbb{R})$ of Hermite functions. Numerical results illustrate the spectral stability in the self-adjoint case. We also notice a strong instability in the non-self-adjoint case, which leads to the computation of 'false eigenvalues' for high energies. In this last case, the resolvent may be very large in norm far from the spectrum.

1.2. Definition of the pseudo-spectra and injectivity pseudo-spectra. - Our interest in this article is to study some notion of semi-classical pseudo-spectrum. In order to justify the definition in the semi-classical setting, we start again with the last example of the rotated harmonic oscillator. Following Zworski in [12], 


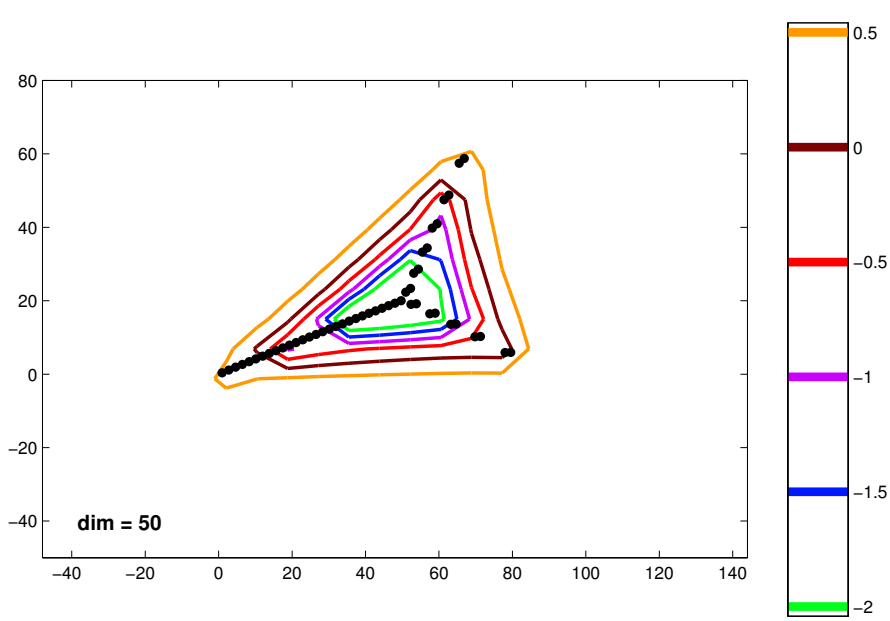

Figure 2. Computation of $\varepsilon$-pseudo-spectra for the rotated harmonic oscillator $P_{\alpha}$ with $\alpha=\frac{\pi}{4}$. The right column gives the corresponding values of $\log _{10} \varepsilon$.

we rephrase the problem of finding eigenvalues for operator $P_{\alpha}$ by a change of scaling. Setting $y=h^{1 / 2} x$ where $h$ is a positive parameter, one has

$$
P_{\alpha}-\lambda=-\frac{d^{2}}{d x^{2}}+e^{i \alpha} x^{2}-\lambda=\frac{1}{h}\left(-h^{2} \frac{d^{2}}{d y^{2}}+e^{i \alpha} y^{2}-h \lambda\right)=\frac{1}{h}\left(P_{\alpha}(h)-z\right)
$$

where $z=h \lambda$ and

$$
P_{\alpha}(h)=-h^{2} \frac{d^{2}}{d y^{2}}+e^{i \alpha} y^{2} .
$$

Since we are interested in the behaviour of the resolvent for large values of $\lambda$, we can work in the semi-classical limit, i.e., $h \rightarrow 0$, with $z$ fixed. We can now extend in a natural way the definition of pseudo-spectrum in the semi-classical setting as follows

DeFINITION 1.2.1. - Let $\left(P_{h}\right)_{0<h \leq 1}$ be a semi-classical family of operators on $L^{2}\left(\mathbb{R}^{n}\right)$ defined on a domain $D$, for all $\mu \geq 0$ the set

$$
\Lambda_{\mu}^{\mathrm{sc}}\left(P_{h}\right)=\left\{z \in \mathbb{C}: \forall C>0, \forall h_{0}>0, \exists 0<h<h_{0},\left\|\left(P_{h}-z\right)^{-1}\right\| \geq C h^{-\mu}\right\},
$$

is called the pseudo-spectrum of index $\mu$ of the family $\left(P_{h}\right)_{0<h \leq 1}$ (we write by convention $\left\|\left(P_{h}-z\right)^{-1}\right\|=+\infty$ if $z$ belongs to the spectrum of $\left.P_{h}\right)$. The pseudo-spectrum of infinite index is defined by

$$
\Lambda_{\infty}^{\mathrm{sc}}\left(P_{h}\right)=\bigcap_{\mu \geq 0} \Lambda_{\mu}^{\mathrm{sc}}\left(P_{h}\right)
$$

TOME $136-2008-\mathrm{N}^{\mathrm{O}} 3$ 
With this definition, the points in the complement of $\Lambda_{\mu}^{\mathrm{sc}}\left(P_{h}\right)$ are the points of the complex plane where we have the following control of the resolvent's norm for $h$ sufficiently small

$$
\exists C>0, \exists h_{0}>0, \forall 0<h<h_{0},\left\|\left(P_{h}-z\right)^{-1}\right\|<C h^{-\mu} .
$$

In this paper, we are interested by the study of slightly different sets from these pseudo-spectra, which are made of points where we can find some quasi-modes with a precise decay in the semi-classical limit. We call these sets the injectivity pseudo-spectra.

DEFINITION 1.2.2. - Let $\left(P_{h}\right)_{0<h \leq 1}$ be a semi-classical family of operators on $L^{2}\left(\mathbb{R}^{n}\right)$ defined on a domain $D$, for all $\mu \geq 0$ the set

$$
\begin{aligned}
\lambda_{\mu}^{\mathrm{sc}}\left(P_{h}\right)=\left\{z \in \mathbb{C}: \forall C>0, \forall h_{0}>0, \exists 0<h<h_{0},\right. & \\
& \left.\exists u \in D,\|u\|_{L^{2}}=1,\left\|\left(P_{h}-z\right) u\right\|_{L^{2}} \leq C h^{\mu}\right\},
\end{aligned}
$$

is called the injectivity pseudo-spectrum of index $\mu$ of the family $\left(P_{h}\right)_{0<h \leq 1}$. The injectivity pseudo-spectrum of infinite index is defined by

$$
\lambda_{\infty}^{\mathrm{sc}}\left(P_{h}\right)=\bigcap_{\mu \geq 0} \lambda_{\mu}^{\mathrm{sc}}\left(P_{h}\right) .
$$

The injectivity pseudo-spectrum of index $\mu$ is by definition the set of points in the complex plane which are some 'almost eigenvalues' with a decay in $O\left(h^{\mu}\right)$ when $h \rightarrow 0$. We notice that the injectivity pseudo-spectra as the pseudo-spectra are non-increasing with the index. The absence of injectivity pseudo-spectrum at a given point is easily characterized by an a priori estimate on the operator. Indeed, there is no injectivity pseudo-spectrum of index $\mu$ at $z$ if and only if we have

$$
\exists C>0, \exists h_{0}>0, \forall 0<h<h_{0}, \forall u \in D,\left\|\left(P_{h}-z\right) u\right\|_{L^{2}} \geq C h^{\mu}\|u\|_{L^{2}} .
$$

We say that there is no loss of any power of $h$, respectively a loss of at most $h^{\mu}$ for the points in the complement of the injectivity pseudo-spectrum of index 0 , respectively of index $\mu$ when $\mu$ is positive. We have the following inclusions

$$
\forall \mu \geq 0, \lambda_{\mu}^{\mathrm{sc}}\left(P_{h}\right) \subset \Lambda_{\mu}^{\mathrm{sc}}\left(P_{h}\right),
$$

but to obtain the equality, we need an additional property of surjectivity for the operators, which is fulfilled for instance if we deal with Fredholm operators of index 0 . We can also notice that if $P_{h}-z$ is a closed operator with a dense domain and that $\bar{z} \notin \lambda_{\mu}^{\mathrm{sc}}\left(P_{h}^{*}\right)$, the estimate (1) for the operator $P_{h}^{*}-\bar{z}$ implies the surjectivity for the operator $P_{h}-z$ if $h$ is sufficiently small. Under these previous assumptions, $z \in \Lambda_{\mu}^{\mathrm{sc}}\left(P_{h}\right)$ implies that $z \in \lambda_{\mu}^{\mathrm{sc}}\left(P_{h}\right)$.

In fact, if we suppose that $P_{h}-z$ is a closed operator, the absence of injectivity pseudo-spectrum in $z$ for the operator $P_{h}$ gives a control for the norm of 
the left inverse $\left(P_{h}-z\right)^{-1}: \operatorname{Ran}\left(P_{h}-z\right) \rightarrow D$ since the estimate (1) induces that the range $\operatorname{Ran}\left(P_{h}-z\right)$ is closed in $L^{2}\left(\mathbb{R}^{n}\right)$. These above definitions differ from one given in [5] for a semi-classical pseudo-differential operator. We prefer here to give a definition, which depends on the properties of the operator rather than on its symbol in order to study some geometrical conditions on the symbol giving the existence or the absence of pseudo-spectrum or injectivity pseudo-spectrum.

1.3. Remark. - The spectrum of a self-adjoint operator is purely real, this property is also true for the injectivity pseudo-spectrum of a family of selfadjoint operators.

Proposition 1.3.1. - Let $\left(P_{h}\right)_{0<h \leq 1}$ be a semi-classical family of selfadjoint operators on $L^{2}\left(\mathbb{R}^{n}\right)$ defined on a dense domain $D$ then

$$
\forall z \in \mathbb{C}, \forall u \in D,\left\|P_{h} u-z u\right\|_{L^{2}} \geq|\operatorname{Im} z|\|u\|_{L^{2}} .
$$

Thus, there is no loss of any power of $h$ in $\mathbb{C} \backslash \mathbb{R}$ and in particular for all $\mu \geq 0$ the injectivity pseudo-spectrum of index $\mu$ of $\left(P_{h}\right)_{0<h \leq 1}$ is contained in $\mathbb{R}$.

Proof. - Let $z$ be in $\mathbb{C} \backslash \mathbb{R}$, the estimate follows from the Cauchy-Schwarz inequality

$$
|\operatorname{Im} z|\|u\|_{L^{2}}^{2} \leq \operatorname{Re}\left(P_{h} u-z u,-i \operatorname{sgn}(\operatorname{Im} z) u\right)_{L^{2}} \leq\left\|P_{h} u-z u\right\|_{L^{2}}\|u\|_{L^{2}},
$$

where $\operatorname{sgn}(x)$ denotes the sign of $x$.

In fact, under the assumptions of the previous proposition we have for all $\mu$ non negative that $\lambda_{\mu}^{\mathrm{sc}}\left(P_{h}\right)=\Lambda_{\mu}^{\mathrm{sc}}\left(P_{h}\right)$. Indeed, we have on one hand by (2) that

$$
\forall z \in \mathbb{C} \backslash \mathbb{R},\left\|\left(P_{h}-z\right)^{-1}\right\| \leq|\operatorname{Im} z|^{-1}
$$

since the spectrum of $P_{h}$ is real by self-adjointness. On the other hand, if $z$ is in $\lambda_{\mu}^{\mathrm{sc}}\left(P_{h}\right)^{c} \cap \mathbb{R}$, a previous remark shows that $z \notin \Lambda_{\mu}^{\mathrm{sc}}\left(P_{h}\right)$ since we have in this case

$$
z=\bar{z} \notin \lambda_{\mu}^{\mathrm{sc}}\left(P_{h}^{*}\right)=\lambda_{\mu}^{\mathrm{sc}}\left(P_{h}\right) .
$$

1.4. Examples. - The first example is the harmonic oscillator

$$
-h^{2} \frac{d^{2}}{d x^{2}}+x^{2}
$$

The injectivity pseudo-spectrum of infinite index is in this case $\mathbb{R}_{+}^{*}$ and one has in the complement in $\mathbb{C}$ of $\mathbb{R}_{+}^{*}$ the following estimates

$$
\begin{gathered}
\forall h>0, \forall u \in C_{0}^{\infty}(\mathbb{R}),\left\|-h^{2} \frac{d^{2} u}{d x^{2}}+x^{2} u\right\|_{L^{2}(\mathbb{R})} \geq h\|u\|_{L^{2}(\mathbb{R})}, \\
\forall z \notin \mathbb{R}_{+}, \exists C>0, \forall h>0, \forall u \in C_{0}^{\infty}(\mathbb{R}),\left\|-h^{2} \frac{d^{2} u}{d x^{2}}+x^{2} u-z u\right\|_{L^{2}(\mathbb{R})} \geq C\|u\|_{L^{2}(\mathbb{R})} .
\end{gathered}
$$

TOME $136-2008-\mathrm{N}^{\circ} 3$ 
For the example of the rotated harmonic oscillator

$$
P_{\alpha}(h)=-h^{2} \frac{d^{2}}{d x^{2}}+e^{i \alpha} x^{2},
$$

with $0<\alpha<\pi$, Davies has proved in [4] (Theorem 1) that

$$
\lambda_{\infty}^{\mathrm{sc}}\left(P_{\alpha}(h)\right) \subset\left\{z \in \mathbb{C}^{*}: 0<\arg z<\alpha\right\} .
$$

In fact, the injectivity pseudo-spectrum of infinite index is exactly

$$
\left\{z \in \mathbb{C}^{*}: 0<\arg z<\alpha\right\} .
$$

Indeed for all $\mu \geq 0, \lambda_{\mu}^{\mathrm{sc}}\left(P_{\alpha}(h)\right)$ is contained in the numerical range

$$
\Sigma\left(P_{\alpha}\right)=\left\{z \in \mathbb{C}^{*}: 0 \leq \arg z \leq \alpha\right\} \cup\{0\} .
$$

We can prove the a priori estimates (see [10])

$$
\forall h>0, \forall u \in C_{0}^{\infty}(\mathbb{R}),\left\|-h^{2} \frac{d^{2} u}{d x^{2}}+e^{i \alpha} x^{2} u\right\|_{L^{2}(\mathbb{R})} \geq \frac{1+\cos \alpha}{2} h\|u\|_{L^{2}(\mathbb{R})},
$$

and for all $z$ in $\mathbb{C}^{*}$ such that $\arg (z) \in\{0, \alpha\}$, there exist some positive constants $C_{z}$ and $h_{0}$ such that

$$
\forall 0<h<h_{0}, \forall u \in C_{0}^{\infty}(\mathbb{R}),\left\|-h^{2} \frac{d^{2} u}{d x^{2}}+e^{i \alpha} x^{2} u-z u\right\|_{L^{2}(\mathbb{R})} \geq C_{z} h^{\frac{2}{3}}\|u\|_{L^{2}(\mathbb{R})} .
$$

\section{Statement of the main result}

2.1. The estimate. - In the following statement, we give an a priori estimate, which characterizes the absence of injectivity pseudo-spectrum in 0 for the semi-classical operator,

$$
h D_{t}+i q(t, x, h \xi)^{w},
$$

where the function $q$ is a real-valued function such that

$$
q(t, x, \xi) \in C_{b}^{2[n / 2]+4}\left(\mathbb{R}_{t} \times \mathbb{R}_{x}^{n} \times \mathbb{R}_{\xi}^{n}, \mathbb{R}\right) .
$$

The notation $C_{b}^{k}$ stands for the space of $C^{k}$ functions, which are bounded as well as their derivatives of order lower than or equal to $k,[m]$ stands for the integer part of $m$ and $q(t, x, h \xi)^{w}$ denotes the Weyl quantization of the symbol $q(t, x, h \xi)$. We assume that for all $X=(x, \xi) \in \mathbb{R}^{2 n}$,

$$
q(t, X)>0 \text { and } s>t \Rightarrow q(s, X) \geq 0 .
$$

This hypothesis means that for all $X \in \mathbb{R}^{2 n}$, the function $t \mapsto q(t, X)$ can only change sign from negative values to positive ones. We also assume that for all $X \in \mathbb{R}^{2 n}$, the function $t \mapsto q(t, X)$ only vanishes in a fixed compact set $[-A, A], A>0$, exactly $N$ times, $N \in \mathbb{N}^{*}$, and that these roots are some 
Lipschitz functions with respect to the variable $X$. More precisely, we assume that

$$
\exists A>0, \inf _{|t| \geq A, X \in \mathbb{R}^{2 n}}|q(t, X)|>0
$$

and

$$
\exists N \in \mathbb{N}^{*}, \forall t \in[-A, A], \forall X \in \mathbb{R}^{2 n}, q(t, X)=e(t, X) \prod_{j=1}^{N}\left(t-\alpha_{j}(X)\right),
$$

where $e$ is a positive function on $\mathbb{R}^{2 n+1}$ such that

$$
M_{0}=\inf _{|t| \leq A, X \in \mathbb{R}^{2 n}} e(t, X)>0
$$

and $\alpha_{j}, j=1, \ldots, N$, are some real-valued Lipschitz functions on $\mathbb{R}^{2 n}$ such that

$$
\left\|\alpha_{j}\right\|_{L^{\infty}\left(\mathbb{R}^{2 n}\right)} \leq A, j=1, \ldots, N .
$$

THEOREM 2.1.1. - Under these assumptions, there exist some constants $C>$ 0 and $0<h_{0} \leq 1$ such that for all $u \in C_{0}^{\infty}\left(\mathbb{R}_{t} \times \mathbb{R}_{x}^{n}\right)$ and $0<h<h_{0}$,

$$
\left\|h D_{t} u+i q(t, x, h \xi)^{w} u\right\|_{L^{2}\left(\mathbb{R}^{n+1}\right)} \geq C h^{\frac{N}{N+1}}\|u\|_{L^{2}\left(\mathbb{R}^{n+1}\right)} .
$$

Thus, there is no injectivity pseudo-spectrum of infinite index in 0. More precisely, there is a loss of at most $h^{N /(N+1)}$ in 0 .

2.2. Remarks. - We can first notice that under the assumptions of Theorem 2.1.1, there is also no injectivity pseudo-spectrum of infinite index in $z$ for all $z$ in $\mathbb{R}$, and that the loss in these points is also of at most $h^{N /(N+1)}$. This fact is a direct consequence of Theorem 2.1.1 and of the following identity

$$
T_{h}\left(h D_{t}+i q(t, x, h \xi)^{w}-z\right) T_{h}^{-1}=h D_{t}+i q(t, x, h \xi)^{w},
$$

where $T_{h}$ is the unitary operator on $L^{2}\left(\mathbb{R}^{n+1}\right)$ defined by

$$
T_{h} u(t, x)=e^{-\frac{2 i \pi}{h} z t} u(t, x) .
$$

Let us now make some comments about the class of pseudo-differential operators we study. The interest of studying such a class is that given a pseudodifferential operator with a principal symbol satisfying the principal-type condition

$$
r(y, \eta)=0 \Rightarrow d r(y, \eta) \neq 0,
$$

we can by multiplication to left and right with some elliptic Fourier integral operators obtain a pseudo-differential operator with a principal symbol, which is microlocally of the type $h D_{t}+i q(t, x, h \xi)^{w}$. To interpret the assumptions of Theorem 2.1.1 in a more general setting, we can notice that the assumption (4) means that the principal symbol satisfies the condition $(\bar{\Psi})$ (see Definition 26.4.6 in [7]). If we make more assumptions of smoothness on the function $e$, 
the assumption (6) implies in terms of iterated Poisson brackets that for all $(t, x, \tau, \xi) \in \mathbb{R}^{2 n+2}$, there exists an integer $0 \leq l \leq N$ such that

$$
H_{\mathrm{Re} p}^{l} \operatorname{Im} p(t, x, \tau, \xi) \neq 0,
$$

if $p(t, x, \tau, \xi)=\tau+i q(t, x, \xi)$. This means that every point in $p\left(\mathbb{R}^{2 n+2}\right)$ are of finite type with an order bounded above by the fixed integer $N$. The assumption (5) of ellipticity outside of the set $[-A, A] \times \mathbb{R}^{2 n}$ allows us to obtain a global subelliptic a priori estimate without conditions on the supports' size for the functions $u$ in $C_{0}^{\infty}\left(\mathbb{R}^{n+1}\right)$. Dencker, Sjöstrand and Zworski have proved in Theorem 1.4 in [5] an absence's result of pseudo-spectrum of infinite index for a general class of pseudo-differential operators. Under the assumptions of Theorem 1.4 in [5], they reduce their study by a symplectic change of variables to the study of the local model $h D_{t}+i q(t, x, h \xi)^{w}$ and prove for this model the a priori estimate (5.9) in [5]. This a priori estimate (5.9) is sufficient to obtain the resolvent's estimate (1.11) in Theorem 1.4 because the assumptions of this theorem 1.4 for getting the a priori estimate (5.9) are also fulfilled for the formal adjoint $h D_{t}-i q(t, x, h \xi)^{w}$, which shows the surjectivity of the operator $h D_{t}+i q(t, x, h \xi)^{w}$ if the domains are suitably chosen and $h$ sufficiently small. In this case, there is no pseudo-spectrum and no injectivity pseudo-spectrum of infinite index in 0 . The loss is of at most $h^{N /(N+1)}$. In the case studied by Dencker, Sjöstrand and Zworski, the condition $(P)$ is fulfilled (see Definition 26.5.1 in [7]). More precisely, in this case the function $q$ does not change sign on $\mathbb{R}^{2 n+1}$. Our result shows that if we are only interested in obtaining the a priori estimate characterizing the absence of injectivity pseudo-spectrum with a loss of at most $h^{N /(N+1)}$, we can obtain a similar a priori estimate as $(5.9)$ for a particular class of pseudo-differential operators violating the condition $(P)$ since we only assume in Theorem 2.1.1 that the condition $(\bar{\Psi})$ is fulfilled.

Another main difference between our result and the result of Dencker, Sjöstrand and Zworski is that we consider here some symbols with limited smoothness. Although our result does not deal with the general subelliptic case (see Proposition 27.6.1 in [7]) in a setting of limited smoothness - indeed, we make a strong assumption of Lipschitz regularity for the roots $\alpha_{j}$ in (6) - we feel that our sharp estimate of the regularity needed for symbols to obtain result of subellipticity is worth noticing and also that it is interesting to have a proof of subelliptic a priori estimates for a class of operators violating the condition $(P)$, which is quite simple in comparison with the proof of the general case given by Hörmander in [7] (Proposition 27.6.1), even if this class is particular and that our result does not deal with the general subelliptic case.

2.3. The structure of the proof. - The first step in the proof of Theorem 2.1.1 is to change the quantization and to prove a similar a priori estimate in another 
quantization. As pointed out before, our assumption on the symbol's sign is essential. To take advantage of this assumption, we use the Wick quantization, which has the main property of being a positive quantization. The next section recalls some results in the Wick quantization, in particular its link with the Weyl quantization. To prove the a priori estimate in the Wick quantization, we need first to use a phase space cut-off to study separately different regions depending on the size of the function $q_{t}^{\prime}$,

$$
h^{\frac{N}{N+1}}\|u\|_{L^{2}}^{2}=h^{\frac{N}{N+1}}\left(H_{1}^{\mathrm{Wick}} u, u\right)_{L^{2}}+h^{\frac{N}{N+1}}\left(H_{2}^{\mathrm{Wick}} u, u\right)_{L^{2}},
$$

where $H_{1}+H_{2}=1, \operatorname{supp} H_{1} \subset\left\{q_{t}^{\prime} \geq \varepsilon_{0}\right\}$ and supp $H_{2} \subset\left\{q_{t}^{\prime} \leq 2 \varepsilon_{0}\right\}, \varepsilon_{0}>0$. The estimate of the first term in the right-hand-side of the previous equality is easier than the second one. We only use for this first term an expansion of a $L^{2}$-norm square and some results of symbolic calculus in the Wick quantization to take advantage of the size of the function $q_{t}^{\prime}$ in Lemma 4.2.1. For the second term, the proof's core of its estimate is the following $L^{2}$-norm splitting

$$
\begin{aligned}
& h^{\frac{N}{N+1}}\left(H_{2}^{\mathrm{Wick}} u, u\right)_{L^{2}}=h^{\frac{N}{N+1}} \int_{\mathbb{R}^{2 n+1}} H_{2}|W u|^{2} d t d X \\
= & h^{\frac{N}{N+1}} \int_{\left\{|q|<h^{N /(N+1)}\right\}} H_{2}|W u|^{2} d t d X+h^{\frac{N}{N+1}} \int_{\left\{|q| \geq h^{N /(N+1)}\right\}} H_{2}|W u|^{2} d t d X,
\end{aligned}
$$

where $W u$ stands for the wave packets transform of $u$ defined in the next section; and we estimate the two terms of the right-hand-side of the following inequality

$$
\begin{aligned}
h^{\frac{N}{N+1}}\left(H_{2}^{\mathrm{Wick}} u, u\right)_{L^{2}} \leq h^{\frac{N}{N+1}} \int_{\left\{|q|<h^{N /(N+1)}\right\}} & H_{2}|W u|^{2} d t d X \\
& +\int_{\mathbb{R}^{2 n+1}} H_{2}|q \| W u|^{2} d t d X
\end{aligned}
$$

in Lemma 4.2.2 and Lemma 4.2.6. To estimate the second term of the righthand-side of (11), we use some techniques developed by Lerner in [8].

\section{Preliminaries}

3.1. Notations and a few facts about the Weyl quantization. - We give in this paragraph the notations and normalizations used in this paper. The scalar product on $L^{2}\left(\mathbb{R}^{n}\right)$ is denoted by

$$
(u, v)_{L^{2}\left(\mathbb{R}^{n}\right)}=\int_{\mathbb{R}^{n}} u(x) \overline{v(x)} d x,
$$

Tоме $136-2008-\mathrm{N}^{\circ} 3$ 
$|\cdot|$ stands for the Euclidean norm and $D_{x}=\partial_{x} /(2 i \pi)$. The definition of the Fourier transform chosen here is, for $u$ in the Schwartz space $\mathcal{S}\left(\mathbb{R}^{n}\right)$,

$$
\widehat{u}(\xi)=\int_{\mathbb{R}^{n}} u(x) e^{-2 i \pi x \cdot \xi} d x,
$$

where $x . \xi$ denotes the canonical scalar product on $\mathbb{R}^{n}$ of $x$ and $\xi$. For a classical Hamiltonian $a(x, \xi)$ defined on $\mathbb{R}_{x}^{n} \times \mathbb{R}_{\xi}^{n}$, the Weyl quantization defines the operator $a^{w}$ by the following formula

$$
\left(a^{w} u\right)(x)=\int_{\mathbb{R}^{2 n}} e^{2 i \pi(x-y) \cdot \xi} a\left(\frac{x+y}{2}, \xi\right) u(y) d y d \xi .
$$

In the statement of Theorem 2.1.1, the variable $t$ is seen as a parameter in the symbol of the operator $q(t, x, h \xi)^{w}$, i.e.,

$$
q(t, x, h \xi)^{w} u(t, x)=\int_{\mathbb{R}^{2 n}} e^{2 i \pi(x-y) \cdot \xi} q\left(t, \frac{x+y}{2}, h \xi\right) u(t, y) d y d \xi
$$

A nice feature of the Weyl quantization is the fact that real Hamiltonians get quantized by (formally) self-adjoint operators. The composition formula in the Weyl quantization, $a^{w} b^{w}=(a \# b)^{w}$, is given by

$$
(a \# b)(X)=2^{2 n} \int_{\mathbb{R}^{4 n}} e^{-4 i \pi \sigma(X-Y, X-Z)} a(Y) b(Z) d Y d Z,
$$

where $\sigma(\cdot, \cdot)$ stands for the symplectic form on $\mathbb{R}^{n} \times \mathbb{R}^{n}$ defined for all $X=(x, \xi)$ and $Y=(y, \eta)$ by $\sigma(X, Y)=\xi . y-\eta . x$.

3.2. Wick calculus. - The purpose of this second paragraph is to recall the definition and some basic properties of the Wick quantization following [9]. We also prove here some results of symbolic calculus we need in the proof of Theorem 2.1.1. The main reason to introduce this new quantization is its property of positivity, i.e., that non-negative Hamiltonians define non-negative operators

$$
q \geq 0 \Rightarrow q^{\text {Wick }} \geq 0 \text {. }
$$

This property of positivity is not satisfied in the case of the Weyl quantization (see [9] for an example of non-negative Hamiltonian defining an operator, which is not non-negative). This property is essential in our approach and permits us to use some sign's hypothesis made on the symbol of studied pseudo-differential operator.

Setting for $x, y$ and $\eta$ in $\mathbb{R}^{n}$,

$$
\varphi_{y, \eta}(x)=2^{n / 4} e^{-\pi(x-y)^{2}} e^{2 i \pi(x-y) \cdot \eta},
$$

where $x^{2}=x_{1}^{2}+\ldots+x_{n}^{2}$, the following lemma introduces the wave packets transform. 
Lemma 3.2.1. - Let $u \in \mathcal{S}\left(\mathbb{R}^{n}\right)$, we define $W u(y, \eta)=\left(u, \varphi_{y, \eta}\right)_{L^{2}\left(\mathbb{R}^{n}\right)}=2^{n / 4} \int_{\mathbb{R}^{n}} u(x) e^{-\pi(x-y)^{2}} e^{-2 i \pi(x-y) \cdot \eta} d x,(y, \eta) \in \mathbb{R}^{2 n}$.

The mapping $u \mapsto W u$ is continuous from $\mathcal{S}\left(\mathbb{R}^{n}\right)$ to $\mathcal{S}\left(\mathbb{R}^{2 n}\right)$ and isometric from $L^{2}\left(\mathbb{R}^{n}\right)$ to $L^{2}\left(\mathbb{R}^{2 n}\right)$. Moreover, we have the reconstruction formula

$$
\forall u \in \mathcal{S}\left(\mathbb{R}^{n}\right), \forall x \in \mathbb{R}^{n}, u(x)=\int_{\mathbb{R}^{2 n}} W u(y, \eta) \varphi_{y, \eta}(x) d y d \eta .
$$

See Lemma 2.1 in [9] for a proof.

Let $Y=(y, \eta) \in \mathbb{R}^{2 n}$, we denote by $\Sigma_{Y}$ the operator defined in the Weyl quantization by the symbol

$$
p_{Y}(X)=2^{n} e^{-2 \pi|X-Y|^{2}} .
$$

This operator is a rank-one orthogonal projection. Indeed, a direct computation gives

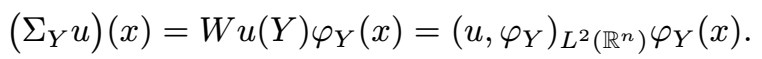

Definition 3.2.1. - Let $a \in L^{\infty}\left(\mathbb{R}^{2 n}\right)$, the Wick quantization of a is defined as

$$
a^{\mathrm{Wick}}=\int_{\mathbb{R}^{2 n}} a(Y) \Sigma_{Y} d Y
$$

REMARK 3.1. - More generally if a belongs to $\mathcal{S}^{\prime}\left(\mathbb{R}^{2 n}\right)$, the operator $a^{\text {Wick }}$ can be defined for all $u$ and $v$ in $\mathcal{S}\left(\mathbb{R}^{n}\right)$ by

$$
<a^{\text {Wick }} u, \bar{v}>_{\mathcal{S}^{\prime}\left(\mathbb{R}^{n}\right), \mathcal{S}\left(\mathbb{R}^{n}\right)}=<a(Y),\left(\Sigma_{Y} u, v\right)_{L^{2}\left(\mathbb{R}^{n}\right)}>_{\mathcal{S}^{\prime}\left(\mathbb{R}^{2 n}\right), \mathcal{S}\left(\mathbb{R}^{2 n}\right)},
$$

where the notation $\langle\cdot, \cdot\rangle_{\mathcal{S}^{\prime}, \mathcal{S}}$ denotes the duality bracket between the spaces $\mathcal{S}^{\prime}$ and $\mathcal{S}$.

Proposition 3.2.1. - Let $a \in L^{\infty}\left(\mathbb{R}^{2 n}\right)$, then

$$
a^{\mathrm{Wick}}=W^{*} a^{\mu} W, 1^{\mathrm{Wick}}=\operatorname{id}_{L^{2}\left(\mathbb{R}^{n}\right)},
$$

where $W$ is the isometric mapping from $L^{2}\left(\mathbb{R}^{n}\right)$ to $L^{2}\left(\mathbb{R}^{2 n}\right)$ defined in Lemma 3.2.1 and $a^{\mu}$ denotes the operator of multiplication by a in $L^{2}\left(\mathbb{R}^{2 n}\right)$. Moreover, one has

$$
\left\|a^{\mathrm{Wick}}\right\|_{\mathcal{L}\left(L^{2}\left(\mathbb{R}^{n}\right)\right)} \leq\|a\|_{L^{\infty}\left(\mathbb{R}^{2 n}\right)} \text { and } a \geq 0 \Rightarrow a^{\mathrm{Wick}} \geq 0 .
$$

See Proposition 3.2 in [9] for a proof.

REMARK 3.2. - We can notice that the previous proposition implies that real Hamiltonians get quantized in the Wick quantization by formally self-adjoint operators. 
Definition 3.2.2. - Let $k \in \mathbb{N}$ and $l \in \mathbb{R}$, the symbol class $S_{k}\left(\Lambda^{l}, \Lambda^{-1} d X^{2}\right)$ denotes the set of $C^{k}$ functions a defined on $\mathbb{R}^{2 n} \times[1,+\infty[$ such that

$$
\forall 0 \leq j \leq k, \gamma_{l, j}(a)=\sup _{\substack{X \in \mathbb{R}^{2 n}, \Lambda \geq 1 \\ T_{p} \in \mathbb{R}^{2 n},\left|T_{p}\right|=1}}\left|\Lambda^{-l+\frac{j}{2}} a^{(j)}(X, \Lambda)\left(T_{1}, \ldots, T_{j}\right)\right|<+\infty
$$

and the symbol class $S\left(\Lambda^{l}, d X^{2}\right)$ denotes the set of $C^{\infty}$ functions a such that

$$
\forall j \in \mathbb{N}, \tilde{\gamma}_{l, j}(a)=\sup _{\substack{X \in \mathbb{R}^{2 n}, \Lambda \geq 1 \\ T_{p} \in \mathbb{R}^{2 n},\left|T_{p}\right|=1}}\left|\Lambda^{-l} a^{(j)}(X, \Lambda)\left(T_{1}, \ldots, T_{j}\right)\right|<+\infty .
$$

Proposition 3.2.2. - Let $a \in L^{\infty}\left(\mathbb{R}^{2 n}\right), b \in S_{2}\left(\Lambda, \Lambda^{-1} d X^{2}\right)$, be some realvalued functions then

$$
\begin{gathered}
a^{\mathrm{Wick}} b^{\mathrm{Wick}}=\left[a b-\frac{1}{4 \pi} a^{\prime} \cdot b^{\prime}+\frac{1}{4 i \pi}\{a, b\}\right]^{\mathrm{Wick}}+S_{1}, \\
\operatorname{Re}\left(a^{\mathrm{Wick}} b^{\mathrm{Wick}}\right)=\left[a b-\frac{1}{4 \pi} a^{\prime} \cdot b^{\prime}\right]^{\mathrm{Wick}}+S_{2},
\end{gathered}
$$

with $\left\|S_{j}\right\|_{\mathcal{L}\left(L^{2}\left(\mathbb{R}^{n}\right)\right)} \leq d_{n}\|a\|_{L^{\infty} \gamma_{1,2}(b), j=1,2 \text {, where the derivatives of a are }}$ taken in the distribution sense, $\{a, b\}$ stands for the Poisson bracket of a and $b$. Here $\gamma_{1,2}(b)$ denotes the semi-norm of $b$ defined in Definition 3.2.2, $d_{n}$ is a positive constant depending only on the dimension $n$ and the distribution $\partial_{X_{j}} a \partial_{X_{l}} b$ is defined as

$$
\partial_{X_{j}} a \partial_{X_{l}} b:=\partial_{X_{j}}\left(a \partial_{X_{l}} b\right)-a \partial_{X_{j}, X_{l}}^{2} b \in \mathcal{S}^{\prime}\left(\mathbb{R}^{2 n}\right)
$$

See Proposition 3.4, its proof and the remark following this proposition in [9] for a proof.

LEMmA 3.2.2. - If $a \in S_{1}\left(\Lambda^{l}, \Lambda^{-1} d X^{2}\right)$, then the Weyl symbol $\tilde{a}$ of $a^{\text {Wick }}$, $a^{\mathrm{Wick}}=\tilde{a}^{w}$, is equal to the function $a * \Gamma$ where $\Gamma(X)=2^{n} e^{-2 \pi|X|^{2}}$, and verifies

$$
\tilde{a} \in S\left(\Lambda^{l}, d X^{2}\right) \text { and } \nabla_{X} \tilde{a} \in S\left(\Lambda^{l-\frac{1}{2}}, d X^{2}\right)
$$

Proof. - From Definition 3.2.1 and (13), one has $a^{\text {Wick }}=\tilde{a}^{w}$ with

$$
\tilde{a}(X)=\int_{\mathbb{R}^{2 n}} a(Y) 2^{n} e^{-2 \pi|X-Y|^{2}} d Y=(a * \Gamma)(X) .
$$

Since for all $\alpha \in \mathbb{N}^{2 n}$,

$$
\partial^{\alpha} \tilde{a}=a * \partial^{\alpha} \Gamma,\left\|\partial^{\alpha} \tilde{a}\right\|_{L^{\infty}} \leq\|a\|_{L^{\infty}}\left\|\partial^{\alpha} \Gamma\right\|_{L^{1}} \text { and }\left\|\partial^{\alpha} \Gamma\right\|_{L^{1}}<+\infty,
$$

we first get that $\tilde{a} \in S\left(\Lambda^{l}, d X^{2}\right)$ because from Definition 3.2.2, one has

$$
\|a\|_{L^{\infty}} \leq \gamma_{l, 0}(a) \Lambda^{l} .
$$


Then, since from Definition 3.2.2, $\nabla_{X} a \in S_{0}\left(\Lambda^{l-\frac{1}{2}}, \Lambda^{-1} d X^{2}\right)$, we deduce from the identity, $\partial_{X}^{\alpha}\left(\nabla_{X} \tilde{a}\right)=\nabla_{X} a * \partial^{\alpha} \Gamma$, using the same estimate as before that

$$
\nabla_{X} \tilde{a} \in S\left(\Lambda^{l-\frac{1}{2}}, d X^{2}\right) .
$$

LEMMA 3.2.3. - If $a \in S\left(\Lambda^{l_{1}}, d X^{2}\right)$ and $b \in S\left(\Lambda^{l_{2}}, d X^{2}\right)$ then the symbol

$$
R(X)=\int_{0}^{1} \int_{\mathbb{R}^{4 n}} e^{-\frac{4 i \pi}{\theta} \sigma(X-Y, X-Z)} a(Y) b(Z) d Y d Z \frac{d \theta}{\theta^{2 n}},
$$

belongs to $S\left(\Lambda^{l_{1}+l_{2}}, d X^{2}\right)$.

Proof. - Setting for $\theta \in] 0,1]$,

$$
R_{\theta}(a, b)(X)=\int_{\mathbb{R}^{4 n}} e^{-\frac{4 i \pi}{\theta} \sigma(X-Y, X-Z)} a(Y) b(Z) \frac{d Y d Z}{\theta^{2 n}},
$$

we deduce from the theorem 18.5.4 in [7] that the bilinear map $(a, b) \mapsto R_{\theta}(a, b)$ from $S\left(\Lambda^{l_{1}}, d X^{2}\right) \times S\left(\Lambda^{l_{2}}, d X^{2}\right)$ to $S\left(\Lambda^{l_{1}+l_{2}}, d X^{2}\right)$ is weakly continuous. Let us assume that $a$ and $b$ belong to the Schwartz space $\mathcal{S}\left(\mathbb{R}^{2 n}\right)$. Using a change of variables, we obtain that

$$
R_{\theta}(a, b)(X)=\int_{\mathbb{R}^{4 n}} e^{-4 i \pi \sigma(Y, Z)} a(\sqrt{\theta} Y+X) b(\sqrt{\theta} Z+X) d Y d Z .
$$

Since an explicit computation gives

$$
\frac{1}{1+|Y|^{4 n+2}+|Z|^{4 n+2}}\left(1+\frac{\left|D_{Y}\right|^{4 n+2}}{2^{4 n+2}}+\frac{\left|D_{Z}\right|^{4 n+2}}{2^{4 n+2}}\right) e^{-4 i \pi \sigma(Y, Z)}=e^{-4 i \pi \sigma(Y, Z)},
$$

we obtain that

$$
\begin{aligned}
& R_{\theta}(a, b)(X)=\int_{\mathbb{R}^{4 n}}\left(1+\frac{\left|D_{Y}\right|^{4 n+2}}{2^{4 n+2}}+\frac{\left|D_{Z}\right|^{4 n+2}}{2^{4 n+2}}\right)\left[e^{-4 i \pi \sigma(Y, Z)}\right] \\
& \times \frac{a(\sqrt{\theta} Y+X) b(\sqrt{\theta} Z+X)}{1+|Y|^{4 n+2}+|Z|^{4 n+2}} d Y d Z
\end{aligned}
$$

and we can make some integrations by parts to obtain that

$$
\begin{aligned}
& R_{\theta}(a, b)(X)= \\
& \int_{\mathbb{R}^{4 n}} e^{-4 i \pi \sigma(Y, Z)}\left(1+\frac{\left|D_{Y}\right|^{4 n+2}}{2^{4 n+2}}+\frac{\left|D_{Z}\right|^{4 n+2}}{2^{4 n+2}}\right)\left[\frac{a(\sqrt{\theta} Y+X) b(\sqrt{\theta} Z+X)}{1+|Y|^{4 n+2}+|Z|^{4 n+2}}\right] d Y d Z .
\end{aligned}
$$

It follows that we can write

$$
\begin{aligned}
& R_{\theta}(a, b)(X)=\int_{\mathbb{R}^{4 n}} \frac{e^{-4 i \pi \sigma(Y, Z)}}{1+|Y|^{4 n+2}+|Z|^{4 n+2}} \\
& \quad \times \sum_{\substack{\alpha, \beta \in \mathbb{N}^{2 n} \\
|\alpha|,|\beta| \leq 4 n+2}} f_{\alpha, \beta}(Y, Z) \sqrt{\theta} \bar{c}^{|\alpha|+|\beta|} \partial^{\alpha} a(\sqrt{\theta} Y+X) \partial^{\beta} b(\sqrt{\theta} Z+X) d Y d Z,
\end{aligned}
$$


where $f_{\alpha, \beta}$ are some $C_{b}^{\infty}\left(\mathbb{R}^{4 n}\right)$ functions. Since $a \in S\left(\Lambda^{l_{1}}, d X^{2}\right), b \in$ $S\left(\Lambda^{l_{2}}, d X^{2}\right)$ and

$$
\int_{\mathbb{R}^{4 n}} \frac{d Y d Z}{1+|Y|^{4 n+2}+|Z|^{4 n+2}}<+\infty
$$

we can differentiate with respect to $X$ the integral of the previous identity and we obtain after these differentiations that for all $\gamma \in \mathbb{N}^{2 n}, X \in \mathbb{R}^{2 n}, \Lambda \geq 1$ and $\theta \in] 0,1]$,

$$
\begin{aligned}
\left|\partial_{X}^{\gamma} R_{\theta}(a, b)(X)\right| \leq(4 n+2)^{4 n} & \left(\int_{\mathbb{R}^{4 n}} \frac{d Y d Z}{1+|Y|^{4 n+2}+|Z|^{4 n+2}}\right) \sup _{\substack{\alpha, \beta \in \mathbb{N}^{2 n} \\
|\alpha|,|\beta| \leq 4 n+2}}\left\|f_{\alpha, \beta}\right\|_{L^{\infty}} \\
& \times \sup _{j \leq 4 n+2+|\gamma|} \tilde{\gamma}_{l_{1}, j}(a) \sup _{j \leq 4 n+2+|\gamma|} \tilde{\gamma}_{l_{2}, j}(b) \Lambda^{l_{1}+l_{2}} .
\end{aligned}
$$

Using the weakly continuity of the map $(a, b) \mapsto R_{\theta}(a, b)$, we deduce from these estimates that the symbol $R_{\theta}(a, b)$ belongs uniformly to the class $S\left(\Lambda^{l_{1}+l_{2}}, d X^{2}\right)$ with respect to $\left.\left.\theta \in\right] 0,1\right]$ if $a \in S\left(\Lambda^{l_{1}}, d X^{2}\right)$ and $b \in S\left(\Lambda^{l_{2}}, d X^{2}\right)$. It follows that $R \in S\left(\Lambda^{l_{1}+l_{2}}, d X^{2}\right)$.

Lemma 3.2.4. - If $a \in S_{1}\left(\Lambda, \Lambda^{-1} d X^{2}\right)$ and $b \in S_{1}\left(1, \Lambda^{-1} d X^{2}\right)$ then there exists a positive constant $C$ such that for all $\Lambda \geq 1$,

$$
\left\|\left[a^{\mathrm{Wick}}, b^{\mathrm{Wick}}\right]\right\|_{\mathcal{L}\left(L^{2}\right)} \leq C,
$$

where $\left[a^{\mathrm{Wick}}, b^{\mathrm{Wick}}\right]$ denotes the commutator of the operators $a^{\mathrm{Wick}}$ and $b^{\mathrm{Wick}}$.

Proof. - We get from Lemma 3.2.2 that

$$
\left[a^{\mathrm{Wick}}, b^{\mathrm{Wick}}\right]=\left[\tilde{a}^{w}, \tilde{b}^{w}\right]=\tilde{a}^{w} \tilde{b}^{w}-\tilde{b}^{w} \tilde{a}^{w}=(\tilde{a} \# \tilde{b}-\tilde{b} \# \tilde{a})^{w},
$$

where $\tilde{a} \in S\left(\Lambda, d X^{2}\right)$ and $\tilde{b} \in S\left(1, d X^{2}\right)$ are some symbols verifying

$$
\nabla_{X} \tilde{a} \in S\left(\Lambda^{\frac{1}{2}}, d X^{2}\right) \text { and } \nabla_{X} \tilde{b} \in S\left(\Lambda^{-\frac{1}{2}}, d X^{2}\right) .
$$

The composition formula (12) and some results of calculus in the Weyl quantization (see the formula following (5) in [1]) show that in the normalization chosen here, one has

$(\tilde{a} \# \tilde{b})(X)=2^{2 n} \int_{\mathbb{R}^{4 n}} e^{-4 i \pi \sigma(X-Y, X-Z)} \tilde{a}(Y) \tilde{b}(Z) d Y d Z=\tilde{a}(X) \tilde{b}(X)+R_{1}(X)$, where

$$
R_{1}(X)=2^{2 n} \int_{0}^{1} \int_{\mathbb{R}^{4 n}} e^{-\frac{4 i \pi}{\theta} \sigma(X-Y, X-Z)} i \pi \sigma\left(D_{Y}, D_{Z}\right)[\tilde{a}(Y) \tilde{b}(Z)] d Y d Z \frac{d \theta}{\theta^{2 n}} .
$$


We deduce from the lemma 3.2.3, (17) and (19) that $R_{1} \in S\left(1, d X^{2}\right)$. In the same way, one has

$$
\tilde{b} \# \tilde{a}=\tilde{a} \tilde{b}+R_{2},
$$

where $R_{2}$ is a symbol in the class $S\left(1, d X^{2}\right)$. It follows from (16), (18) and (20) that

$$
\left[a^{\text {Wick }}, b^{\text {Wick }}\right]=R^{w} \text { with } R=R_{1}-R_{2} \in S\left(1, d X^{2}\right),
$$

which by using the Calderón-Vaillancourt theorem proves the lemma 3.2.4.

LEMmA 3.2.5. - If $a \in S_{2}\left(1, \Lambda^{-1} d X^{2}\right)$ and $b \in S_{2}\left(\Lambda, \Lambda^{-1} d X^{2}\right)$ are some realvalued functions then there exists a positive constant $C$ such that for all $\Lambda \geq 1$,

$$
\left\|a^{\text {Wick }} b^{\text {Wick }} a^{\text {Wick }}-\left(a^{2} b\right)^{\text {Wick }}\right\|_{\mathcal{L}\left(L^{2}\right)} \leq C .
$$

Proof. - We can apply Proposition 3.2.2 to obtain that

$$
a^{\mathrm{Wick}} b^{\mathrm{Wick}}=\left[a b-\frac{1}{4 \pi} a^{\prime} \cdot b^{\prime}+\frac{1}{4 i \pi}\{a, b\}\right]^{\mathrm{Wick}}+S_{1},
$$

where $\left\|S_{1}\right\|_{\mathcal{L}\left(L^{2}\right)} \leq d_{n} \gamma_{0,0}(a) \gamma_{1,2}(b)$ and $d_{n}$ is a positive constant depending only on the dimension $n$. Let us denote

$$
c=-\frac{1}{4 \pi} a^{\prime} \cdot b^{\prime}+\frac{1}{4 i \pi}\{a, b\} .
$$

Since $a \in S_{2}\left(1, \Lambda^{-1} d X^{2}\right)$ and $b \in S_{2}\left(\Lambda, \Lambda^{-1} d X^{2}\right)$, we get that $c \in$ $S_{1}\left(1, \Lambda^{-1} d X^{2}\right)$. It follows from Proposition 3.2.1, (21) and the use of the triangular inequality that

$\left\|c^{\mathrm{Wick}} a^{\mathrm{Wick}}+S_{1} a^{\mathrm{Wick}}\right\|_{\mathcal{L}\left(L^{2}\right)} \leq\left\|c^{\mathrm{Wick}}\right\|_{\mathcal{L}\left(L^{2}\right)}\left\|a^{\mathrm{Wick}}\right\|_{\mathcal{L}\left(L^{2}\right)}+\left\|S_{1}\right\|_{\mathcal{L}\left(L^{2}\right)}\left\|a^{\mathrm{Wick}}\right\|_{\mathcal{L}\left(L^{2}\right)}$

$$
\begin{aligned}
& \leq\|c\|_{L^{\infty}}\|a\|_{L^{\infty}}+d_{n} \gamma_{0,0}(a) \gamma_{1,2}(b)\|a\|_{L^{\infty}} \\
& \leq\left(\gamma_{0,0}(c)+d_{n} \gamma_{0,0}(a) \gamma_{1,2}(b)\right) \gamma_{0,0}(a)<+\infty .
\end{aligned}
$$

Since $\Lambda^{-1} a b \in S_{2}\left(1, \Lambda^{-1} d X^{2}\right)$ and $\Lambda a \in S_{2}\left(\Lambda, \Lambda^{-1} d X^{2}\right)$, another use of Proposition 3.2.2 gives

$$
\begin{aligned}
(a b)^{\text {Wick }} a^{\text {Wick }} & =\left(\Lambda^{-1} a b\right)^{\text {Wick }}(\Lambda a)^{\text {Wick }} \\
& =\left[a^{2} b-\frac{1}{4 \pi}(a b)^{\prime} \cdot a^{\prime}+\frac{1}{4 i \pi}\{a b, a\}\right]^{\text {Wick }}+S_{2},
\end{aligned}
$$

where $\left\|S_{2}\right\|_{\mathcal{L}\left(L^{2}\right)} \leq d_{n} \gamma_{0,0}\left(\Lambda^{-1} a b\right) \gamma_{1,2}(\Lambda a)<+\infty$. According to our assumptions, the symbol

$$
-\frac{1}{4 \pi}(a b)^{\prime} \cdot a^{\prime}+\frac{1}{4 i \pi}\{a b, a\}
$$

TOME $136-2008-\mathrm{N}^{\mathrm{O}} 3$ 
belongs to the class $S_{1}\left(1, \Lambda^{-1} d X^{2}\right)$ and it follows from Proposition 3.2.1 that

$$
\begin{aligned}
\|\left[-(4 \pi)^{-1}(a b)^{\prime} \cdot a^{\prime}+(4 i \pi)^{-1}\right. & \{a b, a\}]^{\mathrm{Wick}} \|_{\mathcal{L}\left(L^{2}\right)} \\
& \leq \gamma_{0,0}\left(-(4 \pi)^{-1}(a b)^{\prime} \cdot a^{\prime}+(4 i \pi)^{-1}\{a b, a\}\right) .
\end{aligned}
$$

Since we have from (21), (22) and (26),

$$
\begin{gathered}
a^{\mathrm{Wick}} b^{\mathrm{Wick}} a^{\mathrm{Wick}}-\left(a^{2} b\right)^{\mathrm{Wick}}=(a b)^{\mathrm{Wick}} a^{\mathrm{Wick}}+c^{\mathrm{Wick}} a^{\mathrm{Wick}}+S_{1} a^{\text {Wick }}-\left(a^{2} b\right)^{\mathrm{Wick}} \\
=\left[-\frac{1}{4 \pi}(a b)^{\prime} \cdot a^{\prime}+\frac{1}{4 i \pi}\{a b, a\}\right]^{\mathrm{Wick}}+S_{2}+c^{\mathrm{Wick}} a^{\mathrm{Wick}}+S_{1} a^{\mathrm{Wick}},
\end{gathered}
$$

we deduce from (23), (26), (28) and the use of the triangular inequality that there exists a positive constant $C$ such that for all $\Lambda \geq 1$,

$$
\left\|a^{\text {Wick }} b^{\text {Wick }} a^{\text {Wick }}-\left(a^{2} b\right)^{\text {Wick }}\right\|_{\mathcal{L}\left(L^{2}\right)} \leq C .
$$

LEMMA 3.2.6. - If $a \in S_{2[n / 2]+4}\left(\Lambda, \Lambda^{-1} d X^{2}\right)$ where $[n / 2]$ stands for the integer part of $n / 2$ then there exists a positive constant $C$ such that for all $\Lambda \geq 1$,

$$
\left\|a^{\text {Wick }}-a^{w}\right\|_{\mathcal{L}\left(L^{2}\right)} \leq C .
$$

Proof. - As in (15), one has $a^{\text {Wick }}=\tilde{a}^{w}$ where

$$
\tilde{a}(X)=\int_{\mathbb{R}^{2 n}} a(Y) 2^{n} e^{-2 \pi|X-Y|^{2}} d Y .
$$

Using a change of variables and a Taylor formula at the second order, we obtain from (29) that

$$
\begin{array}{r}
\tilde{a}(X)=\int_{\mathbb{R}^{2 n}} a(Y+X) 2^{n} e^{-2 \pi|Y|^{2}} d Y=a(X)+\int_{\mathbb{R}^{2 n}} a^{\prime}(X) \cdot Y 2^{n} e^{-2 \pi|Y|^{2}} d Y \\
+\int_{0}^{1} \int_{\mathbb{R}^{2 n}}(1-\theta) a^{\prime \prime}(X+\theta Y) Y^{2} 2^{n} e^{-2 \pi|Y|^{2}} d Y d \theta .
\end{array}
$$

Since

it follows from (30) that

$$
\int_{\mathbb{R}^{2 n}} Y e^{-2 \pi|Y|^{2}} d Y=0
$$

$$
a^{\mathrm{Wick}}-a^{w}=\tilde{a}^{w}-a^{w}=R^{w},
$$

where

$$
R(X)=\int_{0}^{1} \int_{\mathbb{R}^{2 n}}(1-\theta) a^{\prime \prime}(X+\theta Y) Y^{2} 2^{n} e^{-2 \pi|Y|^{2}} d Y d \theta .
$$

Since $a \in S_{2[n / 2]+4}\left(\Lambda, \Lambda^{-1} d X^{2}\right)$ and

$$
\int_{\mathbb{R}^{2 n}}|Y|^{2} e^{-2 \pi|Y|^{2}} d Y<+\infty
$$


we deduce from (32) that $R \in S_{2[n / 2]+2}\left(1, \Lambda^{-1} d X^{2}\right)$ and we can apply the $L^{2}$ estimate for Weyl quantization of Boulkhemair (Theorem 1.2 in [2]) to obtain the existence of a positive constant $C$ such that for all $\Lambda \geq 1$,

$$
\left\|R^{w}\right\|_{\mathcal{L}\left(L^{2}\right)} \leq C .
$$

In view of (31), this ends the proof of Lemma 3.2.6.

\subsection{Another preliminary lemma}

LEMma 3.3.1. - If $F \in C^{1}(\mathbb{R}, \mathbb{C}), \alpha$ is a real-valued Lipschitz function and $G=F \circ \alpha$ then one has

$$
G^{\prime}(x)=F^{\prime}(\alpha(x)) \alpha^{\prime}(x),
$$

for a.e. $x$ in $\mathbb{R}$ if $G^{\prime}$, resp. $\alpha^{\prime}$, stands for the derivative of $G$, resp. $\alpha$, in the distribution sense.

Proof. - To prove this lemma, it is sufficient to show that for all $R>0$, the identity (33) is fulfilled a.e. on ] $-R, R$. Let us consider $R>0$. Since $\alpha^{\prime}$ is a $L^{\infty}(\mathbb{R})$ function because $\alpha$ is a Lipschitz function, we can find a sequence of $C^{0}(\mathbb{R}, \mathbb{R})$ functions $\left(u_{n}\right)_{n \in \mathbb{N}}$ such that for all $n \in \mathbb{N}$,

$$
\left\|u_{n}\right\|_{L^{\infty}([-R, R])} \leq\left\|\alpha^{\prime}\right\|_{L^{\infty}([-R, R])} \text { and } \lim _{n \rightarrow+\infty} u_{n}(x)=\alpha^{\prime}(x),
$$

for a.e. $x$ in $[-R, R]$. We define for all $n \in \mathbb{N}$,

$$
\alpha_{n}(x)=\alpha(0)+\int_{0}^{x} u_{n}(t) d t, x \in \mathbb{R},
$$

and we obtain from (34) that $\alpha_{n}$ is a $C^{1}(\mathbb{R}, \mathbb{R})$ function, which verifies for all $n \in \mathbb{N}$,

$$
\left\|\alpha_{n}^{\prime}\right\|_{L^{\infty}([-R, R])} \leq\left\|\alpha^{\prime}\right\|_{L^{\infty}([-R, R])} \text { and } \lim _{n \rightarrow+\infty} \alpha_{n}^{\prime}(x)=\alpha^{\prime}(x),
$$

for a.e. $x$ in $[-R, R]$. Since we can easily check that the derivative in the distribution sense of the function

$$
\int_{0}^{x} \alpha^{\prime}(t) d t
$$

is equal to $\alpha^{\prime}$, we deduce from the continuity of the function $\alpha$ that for all $x \in \mathbb{R}$,

$$
\alpha(x)=\alpha(0)+\int_{0}^{x} \alpha^{\prime}(t) d t .
$$

Using now (34), (35) and (37), we obtain from the Lebesgue convergence theorem that for all $x \in[-R, R]$,

$$
\lim _{n \rightarrow+\infty} \alpha_{n}(x)=\alpha(x) .
$$

TOME $136-2008-\mathrm{N}^{\mathrm{O}} 3$ 
If $\varphi$ is a $C_{0}^{\infty}(]-R, R[, \mathbb{C})$ function and $\left\langle\cdot, \cdot>_{\mathcal{D}^{\prime}, \mathcal{D}}\right.$ denotes the duality bracket between the distribution space $\mathcal{D}^{\prime}$ and the space of test functions $\mathcal{D}$, one has

$$
\begin{aligned}
<G^{\prime}, \varphi>_{\mathcal{D}^{\prime}, \mathcal{D}}=-<G, \varphi^{\prime}>_{\mathcal{D}^{\prime}, \mathcal{D}} & =-\int_{\mathbb{R}} F(\alpha(x)) \varphi^{\prime}(x) d x \\
& =-\lim _{n \rightarrow+\infty} \int_{\mathbb{R}} F\left(\alpha_{n}(x)\right) \varphi^{\prime}(x) d x,
\end{aligned}
$$

where the last equality is a consequence of the Lebesgue convergence theorem since on one hand, the continuity of $F$ and (38) prove that for all $x \in \mathbb{R}$,

$$
\lim _{n \rightarrow+\infty} F\left(\alpha_{n}(x)\right) \varphi^{\prime}(x)=F(\alpha(x)) \varphi^{\prime}(x),
$$

because $\operatorname{supp} \varphi \subset]-R, R[$ and that on the other hand, one has for all $n \in \mathbb{N}$,

$$
\left|F\left(\alpha_{n}(x)\right) \varphi^{\prime}(x)\right| \leq \sup _{[-M, M]}|F|\left|\varphi^{\prime}(x)\right| \in L^{1},
$$

because it follows from (34), (35) and the use of the triangular inequality that

$$
\left\|\alpha_{n}\right\|_{L^{\infty}([-R, R])} \leq|\alpha(0)|+R\left\|u_{n}\right\|_{L^{\infty}([-R, R])} \leq M,
$$

if $M:=|\alpha(0)|+R\left\|\alpha^{\prime}\right\|_{L^{\infty}(\mathbb{R})}$. We can now deduce from an integration by parts and (39) that

$$
<G^{\prime}, \varphi>_{\mathcal{D}^{\prime}, \mathcal{D}}=\lim _{n \rightarrow+\infty} \int_{\mathbb{R}} F^{\prime}\left(\alpha_{n}(x)\right) \alpha_{n}^{\prime}(x) \varphi(x) d x=\int_{\mathbb{R}} F^{\prime}(\alpha(x)) \alpha^{\prime}(x) \varphi(x) d x,
$$

where the last equality is still a consequence of the Lebesgue convergence theorem since on one hand, one has from (36) and (38),

$$
\lim _{n \rightarrow+\infty} F^{\prime}\left(\alpha_{n}(x)\right) \alpha_{n}^{\prime}(x) \varphi(x)=F^{\prime}(\alpha(x)) \alpha^{\prime}(x) \varphi(x),
$$

for a.e. $x$ in $\mathbb{R}$ because $F^{\prime} \in C^{0}(\mathbb{R}, \mathbb{C})$ and supp $\left.\varphi \subset\right]-R, R[$; and that on the other hand, one has from (36),

$$
\left|F^{\prime}\left(\alpha_{n}(x)\right) \alpha_{n}^{\prime}(x) \varphi(x)\right| \leq \sup _{[-M, M]}\left|F^{\prime}\right|\left\|\alpha^{\prime}\right\|_{L^{\infty}([-R, R])}|\varphi(x)| \in L^{1},
$$

where $M$ is the constant defined in (42). This proves that $G^{\prime}=\left(F^{\prime} \circ \alpha\right) \alpha^{\prime}$ a.e. on $[-R, R]$ and ends the proof of Lemma 3.3.1.

\section{Proof of Theorem 2.1.1}

4.1. A preliminary reduction. - To prove Theorem 2.1.1, it is sufficient to prove the following estimate : there exist some constants $C>0$ and $\Lambda_{0} \geq 1$ such that for all $u \in C_{0}^{\infty}\left(\mathbb{R}^{n+1}\right)$ and $\Lambda \geq \Lambda_{0}$,

$$
\left\|D_{t} u+i \Lambda q\left(t, \Lambda^{-\frac{1}{2}} X\right)^{\mathrm{Wick}} u\right\|_{L^{2}\left(\mathbb{R}^{n+1}\right)} \geq C \Lambda^{\frac{1}{N+1}}\|u\|_{L^{2}\left(\mathbb{R}^{n+1}\right)},
$$


where the variable $t$ is seen as a parameter in the Wick quantization of the symbol $q\left(t, \Lambda^{-\frac{1}{2}} X\right)$ (see Definition 3.2.1),

$$
q\left(t, \Lambda^{-\frac{1}{2}} X\right)^{\mathrm{Wick}}=\int_{\mathbb{R}^{2 n}} q\left(t, \Lambda^{-\frac{1}{2}} X\right) \Sigma_{X} d X .
$$

Indeed, let us assume that the estimate (44) holds and set

$$
Q(t, X, \Lambda)=\Lambda q\left(t, \Lambda^{-\frac{1}{2}} X\right) .
$$

We deduce from (3) and (45) that the function $Q(t, \cdot)$ belongs to the symbol class $S_{2[n / 2]+4}\left(\Lambda, \Lambda^{-1} d X^{2}\right)$ uniformly with respect to the parameter $t$ in $\mathbb{R}$ (see Definition 3.2.2),

$$
Q \in S_{2[n / 2]+4}\left(\Lambda, \Lambda^{-1} d X^{2}\right) .
$$

It follows from Lemma 3.2.6 that there exists a positive constant $c_{0}$ such that for all $\Lambda \geq 1$,

$$
\left\|Q^{\mathrm{Wick}}-Q^{w}\right\|_{\mathcal{L}\left(L^{2}\right)} \leq c_{0}
$$

We deduce from (44), (45), (47) and the use of the triangular inequality that for all $u \in C_{0}^{\infty}\left(\mathbb{R}^{n+1}\right)$ and $\Lambda \geq \Lambda_{0}$,

$$
\begin{aligned}
\left\|D_{t} u+i Q^{w} u\right\|_{L^{2}\left(\mathbb{R}^{n+1}\right)} & \geq\left\|D_{t} u+i Q^{\mathrm{Wick}} u\right\|_{L^{2}\left(\mathbb{R}^{n+1}\right)}-\left\|Q^{\mathrm{Wick}} u-Q^{w} u\right\|_{L^{2}\left(\mathbb{R}^{n+1}\right)} \\
& \geq\left(C \Lambda^{\frac{1}{N+1}}-c_{0}\right)\|u\|_{L^{2}\left(\mathbb{R}^{n+1}\right)} .
\end{aligned}
$$

This estimate induces that there exists $\tilde{\Lambda}_{0} \geq \Lambda_{0}$ such that for all $u \in C_{0}^{\infty}\left(\mathbb{R}^{n+1}\right)$ and $\Lambda \geq \tilde{\Lambda}_{0}$,

$$
\left\|D_{t} u+i \Lambda q\left(t, \Lambda^{-\frac{1}{2}} X\right)^{w} u\right\|_{L^{2}\left(\mathbb{R}^{n+1}\right)} \geq \frac{C}{2} \Lambda^{\frac{1}{N+1}}\|u\|_{L^{2}\left(\mathbb{R}^{n+1}\right)} .
$$

By setting $h_{0}=\tilde{\Lambda}_{0}^{-1}$ and $h=\Lambda^{-1}$, we get from (48) that in the semi-classical setting, one has for all $u \in C_{0}^{\infty}\left(\mathbb{R}^{n+1}\right)$ and $0<h<h_{0}$,

$$
\left\|h D_{t} u+i q\left(t, h^{\frac{1}{2}} X\right)^{w} u\right\|_{L^{2}\left(\mathbb{R}^{n+1}\right)} \geq \frac{C}{2} h^{\frac{N}{N+1}}\|u\|_{L^{2}\left(\mathbb{R}^{n+1}\right)} .
$$

To obtain an analogous estimate for the operator $h D_{t}+i q(t, x, h \xi)^{w}$, we use the following symplectic linear mapping

$$
\chi_{h}(x, \xi)=\left(h^{1 / 2} x, h^{-1 / 2} \xi\right),(x, \xi) \in \mathbb{R}^{2 n} .
$$

Using the symplectic invariance of the Weyl quantization, it follows that

$$
h D_{t}+i q\left(t, h^{1 / 2} x, h^{1 / 2} \xi\right)^{w}=U_{h}^{-1}\left(h D_{t}+i q(t, x, h \xi)^{w}\right) U_{h},
$$

where $U_{h}$ is the unitary operator of $L^{2}\left(\mathbb{R}^{n+1}\right), U_{h} v(t, x)=h^{-\frac{n}{4}} v\left(t, h^{-1 / 2} x\right)$. Eventually, we deduce from (49) and (50) that for all $u \in C_{0}^{\infty}\left(\mathbb{R}^{n+1}\right)$ and $0<h<h_{0}$,

$$
\left\|h D_{t} u+i q(t, x, h \xi)^{w} u\right\|_{L^{2}\left(\mathbb{R}^{n+1}\right)} \geq \frac{C}{2} h^{\frac{N}{N+1}}\|u\|_{L^{2}\left(\mathbb{R}^{n+1}\right)},
$$


which proves the estimate of Theorem 2.1.1.

4.2. Proof of the estimate (44). - To prove the estimate (44), we need to use a phase space cut-off to study separately different regions depending on the size of the function $Q_{t}^{\prime}$. Let us consider $\chi$ a $C^{\infty}(\mathbb{R},[0,1])$ function such that

$$
\chi=0 \text { on }]-\infty, 1], \chi=1 \text { on }[2,+\infty[,
$$

and let us define the following $C^{\infty}(\mathbb{R},[0,1])$ functions

$$
\chi_{1}=\chi^{2} \text { and } \chi_{2}=1-\chi^{2},
$$

which verify

$$
\begin{aligned}
& \left.\left.\chi_{1}+\chi_{2}=1 \text { on } \mathbb{R}, \chi_{1}=0 \text { on }\right]-\infty, 1\right], \chi_{1}=1 \text { on }[2,+\infty[\text { and } \\
& \left.\left.\qquad \chi_{2}=1 \text { on }\right]-\infty, 1\right], \chi_{2}=0 \text { on }[2,+\infty[.
\end{aligned}
$$

Since the functions $\alpha_{j}, j=1, \ldots, N$, appearing in (6) are supposed to be Lipschitzian, we can choose a positive constant $\varepsilon_{0}$ such that

$$
4 N \sup _{j=1, \ldots, N}\left\|\alpha_{j}^{\prime}\right\|_{L^{\infty}\left(\mathbb{R}^{2 n}\right)}^{2} \varepsilon_{0}<1,
$$

where $\alpha_{j}^{\prime}$ stands for the gradient in the distribution sense of the function $\alpha_{j}$ and $\left\|\alpha_{j}^{\prime}\right\|_{L^{\infty}\left(\mathbb{R}^{2 n}\right)}$, the $L^{\infty}$-norm of its Euclidean norm. We define the following symbols

$$
\begin{array}{r}
h_{1}(t, X, \Lambda)=\chi\left(Q_{t}^{\prime}(t, X, \Lambda) \varepsilon_{0}^{-1} \Lambda^{-1}\right), H_{1}(t, X, \Lambda)=\chi_{1}\left(Q_{t}^{\prime}(t, X, \Lambda) \varepsilon_{0}^{-1} \Lambda^{-1}\right) \\
\text { and } H_{2}(t, X, \Lambda)=\chi_{2}\left(Q_{t}^{\prime}(t, X, \Lambda) \varepsilon_{0}^{-1} \Lambda^{-1}\right) .
\end{array}
$$

It follows from (3), (45) and (53) that

$$
H_{1}+H_{2}=1 \text { and } h_{1}, H_{1}, H_{2} \in S_{2[n / 2]+3}\left(1, \Lambda^{-1} d X^{2}\right),
$$

uniformly with respect to the parameter $t \in \mathbb{R}$, and we deduce from (53) and (55) that

$$
\begin{aligned}
& \operatorname{supp} H_{1} \subset\left\{(t, X) \in \mathbb{R}^{2 n+1}: Q_{t}^{\prime}(t, X, \Lambda) \geq \varepsilon_{0} \Lambda\right\} \text { and } \\
& \text { supp } H_{2} \subset\left\{(t, X) \in \mathbb{R}^{2 n+1}: Q_{t}^{\prime}(t, X, \Lambda) \leq 2 \varepsilon_{0} \Lambda\right\} .
\end{aligned}
$$

Step 1. The following lemma gives an estimate of the first term of the righthand-side of (10).

LEMMA 4.2.1. - There exists a positive constant $c_{1}$ such that for all $u \in$ $C_{0}^{\infty}\left(\mathbb{R}^{n+1}\right)$ and $\Lambda \geq 1$,

$$
c_{1} \Lambda\left(H_{1}^{\mathrm{Wick}} u, u\right)_{L^{2}\left(\mathbb{R}^{n+1}\right)} \leq\left\|D_{t} u+i Q^{\mathrm{Wick}} u\right\|_{L^{2}\left(\mathbb{R}^{n+1}\right)}^{2}+\|u\|_{L^{2}\left(\mathbb{R}^{n+1}\right)}^{2} .
$$


Proof of Lemma 4.2.1. - By expanding the following $L^{2}$-norm, we obtain that

$\left\|\left(D_{t}+i Q^{\text {Wick }}\right) h_{1}^{\text {Wick }} u\right\|_{L^{2}}^{2}=\left\|D_{t} h_{1}^{\text {Wick }} u\right\|_{L^{2}}^{2}+2 \operatorname{Re}\left(D_{t} h_{1}^{\text {Wick }} u, i Q^{\text {Wick }} h_{1}^{\text {Wick }} u\right)_{L^{2}}$

$$
\begin{aligned}
+\left\|Q^{\text {Wick }} h_{1}^{\text {Wick }} u\right\|_{L^{2}}^{2} & \geq 2 \operatorname{Re}\left(D_{t} h_{1}^{\text {Wick }} u, i Q^{\text {Wick }} h_{1}^{\text {Wick }} u\right)_{L^{2}} \\
& =\left(\left[D_{t}, i Q^{\text {Wick }}\right] h_{1}^{\text {Wick }} u, h_{1}^{\text {Wick }} u\right)_{L^{2}},
\end{aligned}
$$

since $D_{t}$ and $i Q^{\text {Wick }}$ are respectively formally self-adjoint and anti-self-adjoint operators because $Q$ is a real-valued function (see the remark following the proposition 3.2.1). Since according to (3) and (45), $Q_{t}^{\prime} \in S_{2[n / 2]+3}\left(\Lambda, \Lambda^{-1} d X^{2}\right)$, it follows from Lemma 3.2.5 and (56) that there exists a positive constant $c_{2}$ such that for all $\Lambda \geq 1$,

$$
\left\|h_{1}^{\text {Wick }}\left(Q_{t}^{\prime}\right)^{\text {Wick }} h_{1}^{\text {Wick }}-\left(h_{1}^{2} Q_{t}^{\prime}\right)^{\text {Wick }}\right\|_{\mathcal{L}\left(L^{2}\right)} \leq c_{2} .
$$

Using that $h_{1}^{\text {Wick }}$ is a formally self-adjoint operator because $h_{1}$ is real-valued function, we deduce from the Cauchy-Schwarz inequality and (62) that

$$
\begin{aligned}
& \left(\left[D_{t}, i Q^{\mathrm{Wick}}\right] h_{1}^{\mathrm{Wick}} u, h_{1}^{\mathrm{Wick}} u\right)_{L^{2}}=\frac{1}{2 \pi}\left(\left(Q_{t}^{\prime}\right)^{\mathrm{Wick}} h_{1}^{\mathrm{Wick}} u, h_{1}^{\mathrm{Wick}} u\right)_{L^{2}} \\
= & \frac{1}{2 \pi}\left(h_{1}^{\mathrm{Wick}}\left(Q_{t}^{\prime}\right)^{\mathrm{Wick}} h_{1}^{\mathrm{Wick}} u, u\right)_{L^{2}} \geq \frac{1}{2 \pi}\left(\left(h_{1}^{2} Q_{t}^{\prime}\right)^{\mathrm{Wick}} u, u\right)_{L^{2}}-\frac{c_{2}}{2 \pi}\|u\|_{L^{2}}^{2} .
\end{aligned}
$$

Since from (52) and (55), $h_{1}^{2}=H_{1}$, we obtain from Proposition 3.2.1 and (57) that

$$
\begin{aligned}
& \left(\left(h_{1}^{2} Q_{t}^{\prime}\right)^{\text {Wick }} u, u\right)_{L^{2}\left(\mathbb{R}^{n+1}\right)}=\left(W^{*}\left(h_{1}^{2} Q_{t}^{\prime}\right) W u, u\right)_{L^{2}\left(\mathbb{R}^{n+1}\right)} \\
& =\left(h_{1}^{2} Q_{t}^{\prime} W u, W u\right)_{L^{2}\left(\mathbb{R}^{2 n+1}\right)}=\int_{\mathbb{R}^{2 n+1}} H_{1}(t, X, \Lambda) Q_{t}^{\prime}(t, X, \Lambda)|\Phi(t, X)|^{2} d t d X \\
& \geq \varepsilon_{0} \Lambda \int_{\mathbb{R}^{2 n+1}} H_{1}(t, X, \Lambda)|\Phi(t, X)|^{2} d t d X
\end{aligned}
$$

and

$$
\begin{aligned}
\int_{\mathbb{R}^{2 n+1}} H_{1}(t, X, \Lambda)|\Phi(t, X)|^{2} d t d X=\left(H_{1} W u, W u\right)_{L^{2}\left(\mathbb{R}^{2 n+1}\right)} \\
=\left(W^{*} H_{1} W u, u\right)_{L^{2}\left(\mathbb{R}^{n+1}\right)}=\left(H_{1}^{\text {Wick }} u, u\right)_{L^{2}\left(\mathbb{R}^{n+1}\right)},
\end{aligned}
$$

if $\Phi(t, X)=W(u(t, \cdot))(X)$ where $W$ stands for the wave packets transform in the variable $x$. We deduce from (59), (63) and the two last formulas that

$$
\varepsilon_{0} \Lambda\left(H_{1}^{\mathrm{Wick}} u, u\right)_{L^{2}} \leq 2 \pi\left\|\left(D_{t}+i Q^{\mathrm{Wick}}\right) h_{1}^{\mathrm{Wick}} u\right\|_{L^{2}}^{2}+c_{2}\|u\|_{L^{2}}^{2} .
$$


Using now the proposition 3.2.1 and the triangular inequality, we obtain that (65)

$$
\left\|\left(D_{t}+i Q^{\mathrm{Wick}}\right) h_{1}^{\mathrm{Wick}} u\right\|_{L^{2}}^{2}
$$

$$
\leq 2\left\|h_{1}^{\mathrm{Wick}}\left(D_{t}+i Q^{\mathrm{Wick}}\right) u\right\|_{L^{2}}^{2}+2\left\|\left[D_{t}+i Q^{\mathrm{Wick}}, h_{1}^{\mathrm{Wick}}\right] u\right\|_{L^{2}}^{2}
$$

$$
\begin{aligned}
\leq 2\left\|h_{1}^{\mathrm{Wick}}\right\|_{\mathcal{L}\left(L^{2}\right)}^{2}\left\|D_{t} u+i Q^{\mathrm{Wick}} u\right\|_{L^{2}}^{2}+4 \|\left[D_{t}, h_{1}^{\mathrm{Wick}}\right] & u \|_{L^{2}}^{2} \\
& +4\left\|\left[Q^{\mathrm{Wick}}, h_{1}^{\mathrm{Wick}}\right] u\right\|_{L^{2}}^{2}
\end{aligned}
$$

$$
\begin{aligned}
\leq 2\left\|h_{1}\right\|_{L^{\infty}}^{2}\left\|D_{t} u+i Q^{\mathrm{Wick}} u\right\|_{L^{2}}^{2}+4\left\|\left[D_{t}, h_{1}^{\mathrm{Wick}}\right] u\right\|_{L^{2}}^{2} & \\
& +4\left\|\left[Q^{\mathrm{Wick}}, h_{1}^{\mathrm{Wick}}\right] u\right\|_{L^{2}}^{2},
\end{aligned}
$$

where $[P, Q]$ stands for the commutator of $P$ and $Q$. Using again Proposition 3.2 .1 , we get that

$$
\left\|\left[D_{t}, h_{1}^{\mathrm{Wick}}\right] u\right\|_{L^{2}}=\frac{1}{2 \pi}\left\|\left(\partial_{t} h_{1}\right)^{\mathrm{Wick}} u\right\|_{L^{2}} \leq \frac{1}{2 \pi}\left\|\partial_{t} h_{1}\right\|_{L^{\infty}}\|u\|_{L^{2}} .
$$

Then, we deduce from Lemma 3.2.4, (46) and (56) that there exists a positive constant $c_{3}$ such that for all $\Lambda \geq 1$,

$$
\left\|\left[Q^{\mathrm{Wick}}, h_{1}^{\mathrm{Wick}}\right]\right\|_{\mathcal{L}\left(L^{2}\right)} \leq c_{3} .
$$

Since from (3), (45), (51) and (55),

$$
0 \leq h_{1} \leq 1 \text { and } \partial_{t} h_{1} \in S_{2[n / 2]+2}\left(1, \Lambda^{-1} d X^{2}\right),
$$

uniformly with respect to the parameter $t$ in $\mathbb{R}$, it follows from (65), (69) and (70) that there exists a positive constant $c_{4}$ such that for all $u \in C_{0}^{\infty}\left(\mathbb{R}^{n+1}\right)$ and $\Lambda \geq 1$,

$$
c_{4}\left\|\left(D_{t}+i Q^{\mathrm{Wick}}\right) h_{1}^{\mathrm{Wick}} u\right\|_{L^{2}}^{2} \leq\left\|D_{t} u+i Q^{\mathrm{Wick}} u\right\|_{L^{2}}^{2}+\|u\|_{L^{2}}^{2} .
$$

Then, we get (58) from (64) and (72). This ends the proof of Lemma 4.2.1.

Step 2. In this second step, we estimate the second term of the right-hand-side of (11). This part of the proof uses some techniques developed by Lerner in [8].

LEMMA 4.2.2. - There exists a positive constant $c_{5}$ such that for all $u \in$ $C_{0}^{\infty}\left(\mathbb{R}^{n+1}\right)$ and $\Lambda \geq 1$,

$$
\begin{aligned}
c_{5} \int_{\mathbb{R}^{2 n+1}} H_{2}(t, X, \Lambda) \mid Q(t, X, \Lambda) \| & |(t, X)|^{2} d t d X \\
& \leq\left\|D_{t} u+i Q^{\mathrm{Wick}} u\right\|_{L^{2}}\|u\|_{L^{2}}+\|u\|_{L^{2}}^{2}
\end{aligned}
$$

if $\Phi(t, X)=W(u(t, \cdot))(X)$. 
Proof of Lemma 4.2.2. - For $X \in \mathbb{R}^{2 n}$ and $\Lambda \geq 1$, we define

$$
\theta(X, \Lambda)=\inf \{t \in \mathbb{R}: Q(t, X, \Lambda)>0\},
$$

if the set $\{t \in \mathbb{R}: Q(t, X, \Lambda)>0\}$ is not empty, otherwise we set

$$
\theta(X, \Lambda)=+\infty \text {. }
$$

According to (45), the assumption (4) of Theorem 2.1.1 implies that for all $X \in \mathbb{R}^{2 n}$ and $\Lambda \geq 1$,

$$
Q(t, X, \Lambda)>0 \text { and } s>t \Rightarrow Q(s, X, \Lambda) \geq 0 .
$$

It follows from (74), (75) and (76) that for all $(t, X) \in \mathbb{R}^{2 n+1}$ and $\Lambda \geq 1$, one has

$$
Q(t, X, \Lambda) \operatorname{sgn}(t-\theta(X, \Lambda))=|Q(t, X, \Lambda)|,
$$

where the function sgn is defined by

$$
\operatorname{sgn}(x)=\frac{x}{|x|}, x \in \mathbb{R}^{*}, \operatorname{sgn}(0)=0, \operatorname{sgn}(-\infty)=-1 \text { and } \operatorname{sgn}(+\infty)=1 .
$$

We can now introduce the following multiplier

$$
S(t, X, \Lambda)=\operatorname{sgn}(t-\theta(X, \Lambda)) H_{2}(t, X, \Lambda),(t, X) \in \mathbb{R}^{2 n+1}, \Lambda \geq 1 .
$$

For $u$ in $C_{0}^{\infty}\left(\mathbb{R}^{n+1}\right)$, we obtain by the Cauchy-Schwarz inequality

$$
\begin{aligned}
\left|\operatorname{Re}\left(D_{t} u+i Q^{\mathrm{Wick}} u, i S^{\mathrm{Wick}} u\right)_{L^{2}}\right| \leq \| D_{t} u & +i Q^{\mathrm{Wick}} u\left\|_{L^{2}}\right\| S^{\mathrm{Wick}} u \|_{L^{2}} \\
& \leq\left\|D_{t} u+i Q^{\mathrm{Wick}} u\right\|_{L^{2}}\|u\|_{L^{2}}
\end{aligned}
$$

because it follows from Proposition 3.2.1, (52), (55), (78) and (79) that for all $t \in \mathbb{R}$

$$
\left\|S^{\mathrm{Wick}}(t, \cdot)\right\|_{\mathcal{L}\left(L^{2}\left(\mathbb{R}^{n}\right)\right)} \leq\|S(t, \cdot)\|_{L^{\infty}\left(\mathbb{R}^{2 n}\right)} \leq 1 .
$$

Since $S$ is a real-valued function, $S^{\mathrm{Wick}}$ is a self-adjoint operator and we have

$$
\begin{aligned}
\operatorname{Re}\left(Q^{\mathrm{Wick}} u, S^{\mathrm{Wick}} u\right)_{L^{2}}=\operatorname{Re}\left(S^{\mathrm{Wick}} Q^{\mathrm{Wick}} u, u\right)_{L^{2}} & \\
& =\left(\operatorname{Re}\left(S^{\mathrm{Wick}} Q^{\mathrm{Wick}}\right) u, u\right)_{L^{2}} .
\end{aligned}
$$

Since from (46) and (81), $S \in L^{\infty}$ and $Q \in S_{2[n / 2]+4}\left(\Lambda, \Lambda^{-1} d X^{2}\right)$, we can apply the proposition 3.2.2 to obtain using (81) and (82) that for all $u \in C_{0}^{\infty}\left(\mathbb{R}^{n+1}\right)$ and $\Lambda \geq 1$,

$$
\begin{aligned}
& \text { (83) } \operatorname{Re}\left(D_{t} u+i Q^{\mathrm{Wick}} u, i S^{\mathrm{Wick}} u\right)_{L^{2}\left(\mathbb{R}^{n+1}\right)} \\
& \left.+\left((S Q)^{\mathrm{Wick}} u, u\right)_{L^{2}\left(\mathbb{R}^{n+1}\right)}-\frac{1}{4 \pi}<\left[Q_{X}^{\prime}(t, \cdot) \cdot S_{X}^{\prime}(t, \cdot)\right]^{\mathrm{Wick}} u, \bar{u}>_{\mathcal{S}^{\prime}\left(\mathbb{R}^{n+1}\right), \mathcal{S}\left(\mathbb{R}^{n+1}\right)}^{\mathrm{Wick}} u\right)_{L^{2}\left(\mathbb{R}^{n+1}\right)} \\
& +\int_{\mathbb{R}}(R(t) u(t, \cdot), u(t, \cdot))_{L^{2}\left(\mathbb{R}^{n}\right)} d t,
\end{aligned}
$$

TOME $136-2008-\mathrm{N}^{\mathrm{O}} 3$ 
where for all $t \in \mathbb{R}$,

$$
\|R(t)\|_{\mathcal{L}\left(L^{2}\left(\mathbb{R}^{n}\right)\right)} \leq d_{n}\|S(t, \cdot)\|_{L^{\infty}\left(\mathbb{R}^{2 n}\right)} \gamma_{1,2}(Q) \leq d_{n} \gamma_{1,2}(Q)
$$

and

$$
Q_{X}^{\prime} \cdot S_{X}^{\prime}:=\frac{\partial}{\partial X} \cdot\left(S \frac{\partial Q}{\partial X}\right)-S \operatorname{Trace}\left(Q_{X X}^{\prime \prime}\right) \in \mathcal{S}^{\prime}\left(\mathbb{R}^{2 n+1}\right)
$$

Let us now estimate the four terms of the right-hand-side of (83). Using the Cauchy-Schwarz inequality and the previous estimate, we get for the last one that for all $u \in C_{0}^{\infty}\left(\mathbb{R}^{n+1}\right)$ and $\Lambda \geq 1$,

$$
\left|\int_{\mathbb{R}}(R(t) u(t, \cdot), u(t, \cdot))_{L^{2}\left(\mathbb{R}^{n}\right)} d t\right| \leq d_{n} \gamma_{1,2}(Q)\|u\|_{L^{2}\left(\mathbb{R}^{n+1}\right)}^{2} .
$$

For the first term, we obtain from Proposition 3.2.1 that

$$
\begin{aligned}
& \operatorname{Re}\left(D_{t} u, i S^{\mathrm{Wick}} u\right)_{L^{2}\left(\mathbb{R}^{n+1}\right)}=\operatorname{Re}\left(D_{t} u, i W^{*} S W u\right)_{L^{2}\left(\mathbb{R}^{n+1}\right)} \\
& \quad=\operatorname{Re}\left(D_{t} W u, i S W u\right)_{L^{2}\left(\mathbb{R}^{2 n+1}\right)}=\operatorname{Re}\left(D_{t} \Phi, i S \Phi\right)_{L^{2}\left(\mathbb{R}^{2 n+1}\right)}
\end{aligned}
$$

if $\Phi(t, X)=W(u(t, \cdot))(X)$. A direct computation using (79) and an integration by parts gives

$$
\begin{array}{r}
\operatorname{Re}\left(D_{t} \Phi, i S \Phi\right)_{L^{2}\left(\mathbb{R}^{2 n+1}\right)}=\frac{1}{2 \pi} \int_{\mathbb{R}^{2 n}} H_{2}(\theta(X, \Lambda), X, \Lambda)|\Phi(\theta(X, \Lambda), X)|^{2} d X \\
+\frac{1}{4 \pi} \int_{\mathbb{R}^{2 n+1}} \partial_{t} H_{2}(t, X, \Lambda) \operatorname{sgn}(t-\theta(X, \Lambda))|\Phi(t, X)|^{2} d t d X
\end{array}
$$

Since from (3), (45), (53) and (55), one has for all $(t, X) \in \mathbb{R}^{2 n+1}$ and $\Lambda \geq 1$,

$$
\begin{gathered}
\left|\partial_{t} H_{2}(t, X, \Lambda) \operatorname{sgn}(t-\theta(X, \Lambda))\right|=\varepsilon_{0}^{-1}\left|q_{t t}^{\prime \prime}\left(t, \Lambda^{-\frac{1}{2}} X\right)\right|\left|\chi_{2}^{\prime}\left(Q_{t}^{\prime}(t, X, \Lambda) \varepsilon_{0}^{-1} \Lambda^{-1}\right)\right| \\
\leq \varepsilon_{0}^{-1}\left\|q_{t t}^{\prime \prime}\right\|_{L^{\infty}}\left\|\chi_{2}^{\prime}\right\|_{L^{\infty}}<+\infty,
\end{gathered}
$$

we first obtain that there exists a positive constant $c_{6}$ such that for all $u \in$ $C_{0}^{\infty}\left(\mathbb{R}^{n+1}\right)$ and $\Lambda \geq 1$

$$
\begin{aligned}
& \left.\left|\frac{1}{4 \pi} \int_{\mathbb{R}^{2 n+1}} \partial_{t} H_{2}(t, X, \Lambda) \operatorname{sgn}(t-\theta(X, \Lambda))\right| \Phi(t, X)\right|^{2} d t d X \mid \\
& \leq c_{6}\|\Phi\|_{L^{2}\left(\mathbb{R}^{2 n+1}\right)}^{2}=c_{6}\|W u\|_{L^{2}\left(\mathbb{R}^{2 n+1}\right)}^{2}=c_{6}\|u\|_{L^{2}\left(\mathbb{R}^{n+1}\right)}^{2},
\end{aligned}
$$

because according to Proposition 3.2.1, $W$ is an isometric mapping from $L^{2}\left(\mathbb{R}_{x}^{n}\right)$ to $L^{2}\left(\mathbb{R}_{X}^{2 n}\right)$ and we deduce from (86), (87) and (88) that

$$
\begin{aligned}
& \operatorname{Re}\left(D_{t} u, i S^{\mathrm{Wick}} u\right)_{L^{2}} \geq \\
& \frac{1}{2 \pi} \int_{\mathbb{R}^{2 n}} H_{2}(\theta(X, \Lambda), X, \Lambda)|\Phi(\theta(X, \Lambda), X)|^{2} d X-c_{6}\|u\|_{L^{2}}^{2} .
\end{aligned}
$$


For the second term, we deduce from Proposition 3.2.1 that

$$
\begin{aligned}
& \text { 90) }\left((S Q)^{\mathrm{Wick}} u, u\right)_{L^{2}\left(\mathbb{R}^{n+1}\right)}=\left(W^{*} S Q W u, u\right)_{L^{2}\left(\mathbb{R}^{n+1}\right)} \\
& =(S Q W u, W u)_{L^{2}\left(\mathbb{R}^{2 n+1}\right)}=\int_{\mathbb{R}^{2 n+1}} H_{2}(t, X, \Lambda)|Q(t, X, \Lambda)||\Phi(t, X)|^{2} d t d X,
\end{aligned}
$$

if $\Phi(t, X)=W(u(t, \cdot))(X)$, because according to (77) and (79),

$$
S Q=H_{2} \operatorname{sgn}(t-\theta(X, \Lambda)) Q=H_{2}|Q| .
$$

For the third term, we first deduce using the triangular inequality, (46) and (81) that

$$
\left\|S \operatorname{Trace}\left(Q_{X X}^{\prime \prime}\right)\right\|_{L^{\infty}\left(\mathbb{R}^{2 n+1}\right)} \leq 2 n \gamma_{1,2}(Q) .
$$

Using the Cauchy-Schwarz inequality, the proposition 3.2.1 and (91), we get that for all $u \in C_{0}^{\infty}\left(\mathbb{R}^{n+1}\right)$ and $\Lambda \geq 1$,

$$
\left|<\left[S \operatorname{Trace}\left(Q_{X X}^{\prime \prime}\right)\right]^{\mathrm{Wick}} u, \bar{u}>_{\mathcal{S}^{\prime}, \mathcal{S}}\right|=\left|\left(\left[S \operatorname{Trace}\left(Q_{X X}^{\prime \prime}\right)\right]^{\mathrm{Wick}} u, u\right)_{L^{2}}\right|
$$

$(93) \leq\left\|\left[S \operatorname{Trace}\left(Q_{X X}^{\prime \prime}\right)\right]^{\mathrm{Wick}} u\right\|_{L^{2}}\|u\|_{L^{2}} \leq\left\|\left[S \operatorname{Trace}\left(Q_{X X}^{\prime \prime}\right)\right]^{\mathrm{Wick}}\right\|_{\mathcal{L}\left(L^{2}\right)}\|u\|_{L^{2}}^{2}$

$(94) \leq\left\|S \operatorname{Trace}\left(Q_{X X}^{\prime \prime}\right)\right\|_{L^{\infty}}\|u\|_{L^{2}}^{2} \leq 2 n \gamma_{1,2}(Q)\|u\|_{L^{2}}^{2}$.

In the following, we need to study the distribution

$$
\frac{\partial}{\partial X} \cdot\left(S \frac{\partial Q}{\partial X}\right)
$$

to estimate the term

$$
<\left[\frac{\partial}{\partial X} \cdot\left(S \frac{\partial Q}{\partial X}\right)\right]^{\text {Wick }} u, \bar{u}>_{\mathcal{S}^{\prime}\left(\mathbb{R}^{n+1}\right), \mathcal{S}\left(\mathbb{R}^{n+1}\right)} .
$$

Let $\chi_{0}: \mathbb{R} \rightarrow[0,1]$ be a $C^{\infty}$ function such that

$$
\chi_{0}=1 \text { on }[-1,1] \text { and supp } \chi_{0} \subset[-2,2] .
$$

Setting

$$
\tau_{0}=\frac{\partial}{\partial X} \cdot\left(\chi_{0}\left(\left|Q_{X}^{\prime}\right|^{2}\right) S \frac{\partial Q}{\partial X}\right) \text { and } \tau_{1}=\frac{\partial}{\partial X} \cdot\left(w_{0}\left(\left|Q_{X}^{\prime}\right|^{2}\right) S \frac{\partial Q}{\partial X}\right)
$$

where $w_{0}=1-\chi_{0}$, one has

$$
\frac{\partial}{\partial X} \cdot\left(S \frac{\partial Q}{\partial X}\right)=\tau_{0}+\tau_{1},
$$

with $\tau_{0} \in \mathcal{S}^{\prime}\left(\mathbb{R}^{2 n+1}\right)$ and $\tau_{1} \in \mathcal{S}^{\prime}\left(\mathbb{R}^{2 n+1}\right)$. 
Estimate of $\left\langle\tau_{0}^{\text {Wick }} u, \bar{u}>_{\mathcal{S}^{\prime}, \mathcal{S}}\right.$. We deduce from (97) and the remark following the definition 3.2.1 that

$$
\begin{aligned}
& \quad=\int_{\mathbb{R}}<\frac{\partial}{\partial X} \cdot\left(\chi_{0}\left(\left|Q_{X}^{\prime}\right|^{2}\right) S \frac{\partial Q}{\partial X}\right),\left(\Sigma_{Y} u(t, \cdot), u(t, \cdot)\right)_{L^{2}\left(\mathbb{R}^{n}\right)}>_{\mathcal{S}^{\prime}\left(\mathbb{R}_{Y}^{2 n}\right), \mathcal{S}\left(\mathbb{R}_{Y}^{2 n}\right)} d t \\
& \quad=-\int_{\mathbb{R}^{2 n+1}} \chi_{0}\left(\left|Q_{X}^{\prime}\right|^{2}\right) S \frac{\partial Q}{\partial X} \cdot \frac{\partial}{\partial X}\left[\left(\Sigma_{X} u(t, \cdot), u(t, \cdot)\right)_{L^{2}\left(\mathbb{R}^{n}\right)}\right] d t d X .
\end{aligned}
$$

To evaluate (99), we need to use the following lemma.

Lemma 4.2.3. - There exists a constant $D_{n}$ depending only on the dimension such that for all $\alpha$ in $L^{\infty}\left(\mathbb{R}^{2 n}\right)$ and for all $j=1, \ldots, 2 n$,

$$
\left\|\int_{\mathbb{R}^{2 n}} \alpha(Y) \frac{\partial}{\partial Y_{j}}\left(\Sigma_{Y}\right) d Y\right\|_{\mathcal{L}\left(L^{2}\left(\mathbb{R}^{n}\right)\right)} \leq D_{n}\|\alpha\|_{L^{\infty}\left(\mathbb{R}^{2 n}\right)} .
$$

See (6.10) in [8] for a proof of this lemma.

Since from (96), supp $\chi_{0} \subset[-2,2]$, we notice from (81) that one has

$$
\left\|\chi_{0}\left(\left|Q_{X}^{\prime}\right|^{2}\right) S\left|Q_{X}^{\prime}\right|\right\|_{L^{\infty}} \leq \sqrt{2}\left\|\chi_{0}\right\|_{L^{\infty}}
$$

Then, using successively the triangular inequality, the Cauchy-Schwarz inequality, Lemma 4.2.3 and (102), we deduce that there exists a positive constant $c_{7}$ such that for all $u \in C_{0}^{\infty}\left(\mathbb{R}^{2 n+1}\right)$ and $\Lambda \geq 1$,

$$
=\left|\left(\left[\int_{\mathbb{R}^{2 n}} \chi_{0}\left(\left|Q_{X}^{\prime}(t, \cdot)\right|^{2}\right) S(t, \cdot) \frac{\partial Q}{\partial X}(t, \cdot) \cdot \frac{\partial}{\partial X}\left(\Sigma_{X}\right) d X\right] u, u\right)_{L^{2}\left(\mathbb{R}^{n+1}\right)}\right|
$$

$$
\leq \sum_{j=1}^{2 n}\left|\left(\left[\int_{\mathbb{R}^{2 n}} \chi_{0}\left(\left|Q_{X}^{\prime}(t, \cdot)\right|^{2}\right) S(t, \cdot) \frac{\partial Q}{\partial X_{j}}(t, \cdot) \frac{\partial}{\partial X_{j}}\left(\Sigma_{X}\right) d X\right] u, u\right)_{L^{2}\left(\mathbb{R}^{n+1}\right)}\right|
$$


(106)

$$
\leq \sum_{j=1}^{2 n}\left\|\int_{\mathbb{R}^{2 n}} \chi_{0}\left(\left|Q_{X}^{\prime}(t, \cdot)\right|^{2}\right) S(t, \cdot) \frac{\partial Q}{\partial X_{j}}(t, \cdot) \frac{\partial}{\partial X_{j}}\left(\Sigma_{X}\right) d X\right\|_{\mathcal{L}\left(L^{2}\right)}\|u\|_{L^{2}\left(\mathbb{R}^{n+1}\right)}^{2}
$$

$$
\leq c_{7}\|u\|_{L^{2}}^{2} \text {. }
$$

Eventually, we deduce from (99) and (103) that for all $u \in C_{0}^{\infty}\left(\mathbb{R}^{n+1}\right)$ and $\Lambda \geq 1$

$$
\left|<\tau_{0}^{\text {Wick }} u, \bar{u}>_{\mathcal{S}^{\prime}\left(\mathbb{R}^{n+1}\right), \mathcal{S}\left(\mathbb{R}^{n+1}\right)}\right| \leq c_{7}\|u\|_{L^{2}\left(\mathbb{R}^{n+1}\right)}^{2} .
$$

Estimate of $<\tau_{1}^{\mathrm{Wick}} u, \bar{u}>_{\mathcal{S}^{\prime}, \mathcal{S}}$. Let us set

$$
\tilde{S}(t, X, \Lambda)=\operatorname{sgn}(t-\theta(X, \Lambda))
$$

We get from (79) that $S=H_{2} \tilde{S}$, and we deduce from (97) and the fact that $\tilde{S}$ is a zero order distribution that

$$
\tau_{1}=\frac{\partial}{\partial X} \cdot\left(w_{0}\left(\left|Q_{X}^{\prime}\right|^{2}\right) H_{2} \frac{\partial Q}{\partial X}\right) \tilde{S}+w_{0}\left(\left|Q_{X}^{\prime}\right|^{2}\right) H_{2} \frac{\partial Q}{\partial X} \cdot \frac{\partial \tilde{S}}{\partial X} .
$$

Since from (96) and (97), the support of the function $w_{0}^{\prime}$ is contained in $[-2,2]$ and $0 \leq w_{0} \leq 1$, we get using the triangular inequality, (46) and (56) that for all $(t, X) \in \mathbb{R}^{2 n+1}$ and $\Lambda \geq 1$,

$$
\begin{aligned}
& \left|\frac{\partial}{\partial X} \cdot\left(w_{0}\left(\left|Q_{X}^{\prime}\right|^{2}\right) H_{2} \frac{\partial Q}{\partial X}\right)\right| \leq 2\left|w_{0}^{\prime}\left(\left|Q_{X}^{\prime}\right|^{2}\right) H_{2} Q_{X X}^{\prime \prime}\left(Q_{X}^{\prime}, Q_{X}^{\prime}\right)\right| \\
& +\left|w_{0}\left(\left|Q_{X}^{\prime}\right|^{2}\right) H_{2} \operatorname{Trace}\left(Q_{X X}^{\prime \prime}\right)\right|+\left|w_{0}\left(\left|Q_{X}^{\prime}\right|^{2}\right) \frac{\partial H_{2}}{\partial X} \cdot \frac{\partial Q}{\partial X}\right| \\
& \leq 4\left\|w_{0}^{\prime}\right\|_{L^{\infty}} \gamma_{0,0}\left(H_{2}\right) \gamma_{1,2}(Q)+2 n\left\|w_{0}\right\|_{L^{\infty}} \gamma_{0,0}\left(H_{2}\right) \gamma_{1,2}(Q) \\
& +\left\|w_{0}\right\|_{L^{\infty}} \gamma_{0,1}\left(H_{2}\right) \gamma_{1,1}(Q)<+\infty \text {. }
\end{aligned}
$$

It follows from (109) and (111) that there exists a positive constant $c_{8}$ such that for all $\Lambda \geq 1$,

$$
\left\|\frac{\partial}{\partial X} \cdot\left(w_{0}\left(\left|Q_{X}^{\prime}\right|^{2}\right) H_{2} \frac{\partial Q}{\partial X}\right) \tilde{S}\right\|_{L^{\infty}\left(\mathbb{R}^{2 n+1}\right)} \leq c_{8}
$$

TOME $136-2008-\mathrm{N}^{\mathrm{O}} 3$ 
We deduce using the Cauchy-Schwarz inequality, Proposition 3.2.1 and (115) that for all $u \in C_{0}^{\infty}\left(\mathbb{R}^{2 n+1}\right)$ and $\Lambda \geq 1$,

$(117)=\left|\left(\left[\frac{\partial}{\partial X} \cdot\left(w_{0}\left(\left|Q_{X}^{\prime}\right|^{2}\right) H_{2} \frac{\partial Q}{\partial X}\right) \tilde{S}\right]^{\text {Wick }} u, u\right)_{L^{2}\left(\mathbb{R}^{n+1}\right)}\right|$

$(118) \leq\left\|\left[\frac{\partial}{\partial X} \cdot\left(w_{0}\left(\left|Q_{X}^{\prime}\right|^{2}\right) H_{2} \frac{\partial Q}{\partial X}\right) \tilde{S}\right]^{\text {Wick }} u\right\|_{L^{2}\left(\mathbb{R}^{n+1}\right)}\|u\|_{L^{2}\left(\mathbb{R}^{n+1}\right)}$

$(119) \leq\left\|\left[\frac{\partial}{\partial X} \cdot\left(w_{0}\left(\left|Q_{X}^{\prime}\right|^{2}\right) H_{2} \frac{\partial Q}{\partial X}\right) \tilde{S}\right]^{\text {Wick }}\right\|_{\mathcal{L}\left(L^{2}\right)}\|u\|_{L^{2}\left(\mathbb{R}^{n+1}\right)}^{2}$

$(120) \leq\left\|\frac{\partial}{\partial X} \cdot\left(w_{0}\left(\left|Q_{X}^{\prime}\right|^{2}\right) H_{2} \frac{\partial Q}{\partial X}\right) \tilde{S}\right\|_{L^{\infty}\left(\mathbb{R}^{2 n+1}\right)}\|u\|_{L^{2}\left(\mathbb{R}^{n+1}\right)}^{2} \leq c_{8}\|u\|_{L^{2}\left(\mathbb{R}^{n+1}\right)}^{2}$.

To study the second term in the right-hand-side of (110), we first prove the following lemma.

LEMMA 4.2.4. - One has the following inclusions

$$
\operatorname{supp} \tilde{S}_{X}^{\prime} \subset\left\{(t, X) \in \mathbb{R}^{2 n+1}: Q(t, X, \Lambda)=0\right\}
$$

and

(121) $\operatorname{supp}\left(w_{0}\left(\left|Q_{X}^{\prime}\right|^{2}\right) H_{2} \frac{\partial Q}{\partial X} \cdot \frac{\partial \tilde{S}}{\partial X}\right) \subset$

$K=\left\{(t, X) \in \mathbb{R}^{2 n+1}: Q(t, X, \Lambda)=0,\left|Q_{X}^{\prime}(t, X, \Lambda)\right| \geq 1\right.$ and $\left.Q_{t}^{\prime}(t, X, \Lambda) \leq 2 \varepsilon_{0} \Lambda\right\}$.

Moreover, the distribution $\delta(Q) w_{0}\left(\left|Q_{X}^{\prime}\right|^{2}\right)$ is well defined on $\mathbb{R}^{2 n+1}$ and one has

$$
w_{0}\left(\left|Q_{X}^{\prime}\right|^{2}\right) H_{2} \frac{\partial Q}{\partial X} \cdot \frac{\partial \tilde{S}}{\partial X}=2 \delta(Q)\left|Q_{X}^{\prime}\right|^{2} w_{0}\left(\left|Q_{X}^{\prime}\right|^{2}\right) H_{2}
$$

Proof of lemma 4.2.4. - Since from (77) and (109), $\tilde{S}=1$ on $\{Q>0\}$ and $\tilde{S}=-1$ on $\{Q<0\}$, the support of $\tilde{S}_{X}^{\prime}$ is included in $\left\{(t, X) \in \mathbb{R}^{2 n+1}\right.$ : $Q(t, X, \Lambda)=0\}$. Moreover, since from (96) and (97), supp $\left.\left.w_{0} \subset\right]-\infty,-1\right] \cup$ $[1,+\infty[$, and from (57),

$$
\text { supp } H_{2} \subset\left\{(t, X) \in \mathbb{R}^{2 n+1}: Q_{t}^{\prime}(t, X, \Lambda) \leq 2 \varepsilon_{0} \Lambda\right\},
$$

we deduce that the support of the distribution

$$
w_{0}\left(\left|Q_{X}^{\prime}\right|^{2}\right) H_{2} \frac{\partial Q}{\partial X} \cdot \frac{\partial \tilde{S}}{\partial X}
$$

verifies the inclusion (121). Since from (5), (6), (7), (8) and (45), one has

$$
\left\{(t, X) \in \mathbb{R}^{2 n+1}: Q(t, X, \Lambda)=0\right\}=\bigcup_{j=1}^{N}\left\{\left(\alpha_{j}\left(\Lambda^{-\frac{1}{2}} X\right), X\right): X \in \mathbb{R}^{2 n}\right\},
$$


it follows that for all $\Lambda \geq 1$ the Lebesgue measure in $\mathbb{R}^{2 n+1}$ of the set

$$
\left\{(t, X) \in \mathbb{R}^{2 n+1}: Q(t, X, \Lambda)=0\right\},
$$

is zero and we get from (77) and (109) that $\tilde{S}$ is equal to the $L^{\infty}$ function $Q /|Q|$ and

$$
w_{0}\left(\left|Q_{X}^{\prime}\right|^{2}\right) H_{2} \frac{\partial Q}{\partial X} \cdot \frac{\partial \tilde{S}}{\partial X}=w_{0}\left(\left|Q_{X}^{\prime}\right|^{2}\right) H_{2} \frac{\partial Q}{\partial X} \cdot \frac{\partial}{\partial X}\left(\frac{Q}{|Q|}\right) .
$$

We denote by $\delta(Q)$ the pullback of $\delta_{0}$ the Dirac measure at 0 in $\mathcal{D}^{\prime}(\mathbb{R})$ by $Q$. This distribution $\delta(Q)=Q^{*} \delta_{0}$ is well defined on

$$
\Omega=\left\{(t, X) \in \mathbb{R}^{2 n+1}:\left|Q_{X}^{\prime}(t, X, \Lambda)\right|>0\right\}
$$

and one has on $\Omega$,

$$
\frac{\partial}{\partial X}\left(\frac{Q}{|Q|}\right)=\frac{\partial}{\partial X}\left(Q^{*} \operatorname{sgn}\right)=\frac{\partial Q}{\partial X} Q^{*}(\partial \operatorname{sgn})=2 \frac{\partial Q}{\partial X} \delta(Q),
$$

which induces from (122) that

$$
w_{0}\left(\left|Q_{X}^{\prime}\right|^{2}\right) H_{2} \frac{\partial Q}{\partial X} \cdot \frac{\partial \tilde{S}}{\partial X}=2 \delta(Q)\left|Q_{X}^{\prime}\right|^{2} w_{0}\left(\left|Q_{X}^{\prime}\right|^{2}\right) H_{2},
$$

on $\Omega$. Since from (96), (97) and (123),

$$
\operatorname{supp} w_{0}\left(\left|Q_{X}^{\prime}\right|^{2}\right) \subset \Omega,
$$

we deduce that the distribution $2 \delta(Q)\left|Q_{X}^{\prime}\right|^{2} w_{0}\left(\left|Q_{X}^{\prime}\right|^{2}\right) H_{2}$ is well defined on $\mathbb{R}^{2 n+1}$ and that the identity (124) is fulfilled on $\mathbb{R}^{2 n+1}$.

We deduce now from the remark following the definition 3.2.1 and Lemma 4.2.4 that

$$
<\left[w_{0}\left(\left|Q_{X}^{\prime}\right|^{2}\right) H_{2} \frac{\partial Q}{\partial X} \cdot \frac{\partial \tilde{S}}{\partial X}\right]^{\text {Wick }} u, \bar{u}>_{\mathcal{S}^{\prime}\left(\mathbb{R}^{n+1}\right), \mathcal{S}\left(\mathbb{R}^{n+1}\right)}
$$

$$
=<w_{0}\left(\left|Q_{X}^{\prime}\right|^{2}\right) H_{2} \frac{\partial Q}{\partial X} \cdot \frac{\partial \tilde{S}}{\partial X},\left(\Sigma_{X} u(t, \cdot), u(t, \cdot)\right)_{L^{2}\left(\mathbb{R}^{n}\right)}>_{\mathcal{S}^{\prime}\left(\mathbb{R}^{2 n+1}\right), \mathcal{S}\left(\mathbb{R}^{2 n+1}\right)}
$$

$$
=<2 \delta(Q)\left|Q_{X}^{\prime}\right|^{2} w_{0}\left(\left|Q_{X}^{\prime}\right|^{2}\right) H_{2},|\Phi|^{2}>_{\mathcal{S}^{\prime}\left(\mathbb{R}^{2 n+1}\right), \mathcal{S}\left(\mathbb{R}^{2 n+1}\right)}
$$

because $\left(\Sigma_{X} u(t, \cdot), u(t, \cdot)\right)_{L^{2}\left(\mathbb{R}^{n}\right)}=|\Phi(t, X)|^{2}$ according to Lemma 3.2.1 and (14) if $\Phi(t, X)=W(u(t, \cdot))(X)$. To estimate (126), we need to prove the following lemma.

Lemma 4.2.5. - For all $\Lambda \geq 1$ and $(t, X) \in\left\{Q=0\right.$ and $\left.\left|Q_{X}^{\prime}\right|>1 / 2\right\}$, we have

$$
\frac{1}{2}<\left|Q_{X}^{\prime}(t, X, \Lambda)\right| \leq \Lambda^{-\frac{1}{2}} \sup _{j=1, \ldots, N}\left\|\alpha_{j}^{\prime}\right\|_{L^{\infty}\left(\mathbb{R}^{2 n}\right)} Q_{t}^{\prime}(t, X, \Lambda) .
$$


Proof of Lemma 4.2.5. - Let $\left(t_{0}, X_{0}\right) \in \mathbb{R}^{2 n+1}$ such that

$$
Q\left(t_{0}, X_{0}, \Lambda\right)=0 \text { and }\left|Q_{X}^{\prime}\left(t_{0}, X_{0}, \Lambda\right)\right|>\frac{1}{2} \text {. }
$$

It follows from (5), (6), (7) and (45) that there exists $j_{0} \in\{1, \ldots, N\}$ such that

$$
t_{0}=\alpha_{j_{0}}\left(\Lambda^{-\frac{1}{2}} X_{0}\right)
$$

Since from (6), (8) and (45), one has for all $j \in\{1, \ldots, N\}, X \in \mathbb{R}^{2 n}$ and $\Lambda \geq 1$,

$$
Q\left(\alpha_{j}\left(\Lambda^{-\frac{1}{2}} X\right), X, \Lambda\right)=0
$$

we obtain from Lemma 3.3.1 that

$$
Q_{t}^{\prime}\left(\alpha_{j}\left(\Lambda^{-\frac{1}{2}} X\right), X, \Lambda\right) \alpha_{j}^{\prime}\left(\Lambda^{-\frac{1}{2}} X\right) \Lambda^{-\frac{1}{2}}+Q_{X}^{\prime}\left(\alpha_{j}\left(\Lambda^{-\frac{1}{2}} X\right), X, \Lambda\right)=0
$$

for a.e. $X$ in $\mathbb{R}^{2 n}$, which induces that

$$
\left|Q_{X}^{\prime}\left(\alpha_{j}\left(\Lambda^{-\frac{1}{2}} X\right), X, \Lambda\right)\right| \leq \Lambda^{-\frac{1}{2}} \sup _{j=1, \ldots, N}\left\|\alpha_{j}^{\prime}\right\|_{L^{\infty}\left(\mathbb{R}^{2 n}\right)}\left|Q_{t}^{\prime}\left(\alpha_{j}\left(\Lambda^{-\frac{1}{2}} X\right), X, \Lambda\right)\right|,
$$

for a.e. $X$ in $\mathbb{R}^{2 n}$. Then, we first notice that the continuity of the functions in the previous estimate proves that in fact this estimate is fulfilled for all $X \in \mathbb{R}^{2 n}$. We obtain from (129), (130) and (133) that

$$
\frac{1}{2}<\left|Q_{X}^{\prime}\left(t_{0}, X_{0}, \Lambda\right)\right| \leq \Lambda^{-\frac{1}{2}} \sup _{j=1, \ldots, N}\left\|\alpha_{j}^{\prime}\right\|_{L^{\infty}\left(\mathbb{R}^{2 n}\right)}\left|Q_{t}^{\prime}\left(t_{0}, X_{0}, \Lambda\right)\right| .
$$

To end the proof of this lemma, it is sufficient to check that $Q_{t}^{\prime}\left(t_{0}, X_{0}, \Lambda\right) \geq 0$. This is the case because if $Q_{t}^{\prime}\left(t_{0}, X_{0}, \Lambda\right)<0$, we would deduce from (45) and (129) that the function

$$
t \mapsto q\left(t, \Lambda^{-\frac{1}{2}} X_{0}\right)
$$

would change sign from positive values to negative ones at the first order in $t_{0}$, which is not possible in view of the assumption (4).

Since from (52), (55), (96) and (97),

$$
\left.\left.0 \leq H_{2} \leq 1,0 \leq w_{0} \leq 1 \text { and } \operatorname{supp} w_{0} \subset\right]-\infty,-1\right] \cup[1,+\infty[,
$$

we deduce from Lemma 4.2.5 that

$$
\begin{aligned}
0 & \leq<2 \delta(Q)\left|Q_{X}^{\prime}\right|^{2} w_{0}\left(\left|Q_{X}^{\prime}\right|^{2}\right) H_{2},|\Phi|^{2}>_{\mathcal{S}^{\prime}, \mathcal{S}} \\
& \leq 2 \Lambda^{-1} \sup _{j=1, \ldots, N}\left\|\alpha_{j}^{\prime}\right\|_{L^{\infty}}^{2}<\delta(Q)\left(Q_{t}^{\prime}\right)^{2} w_{0}\left(\left|Q_{X}^{\prime}\right|^{2}\right) H_{2},|\Phi|^{2}>_{\mathcal{S}^{\prime}, \mathcal{S}} \\
& \leq 4 \varepsilon_{0} \sup _{j=1, \ldots, N}\left\|\alpha_{j}^{\prime}\right\|_{L^{\infty}}^{2}<\delta(Q) Q_{t}^{\prime} w_{0}\left(\left|Q_{X}^{\prime}\right|^{2}\right) H_{2},|\Phi|^{2}>_{\mathcal{S}^{\prime}, \mathcal{S}},
\end{aligned}
$$

because according to (57), $Q_{t}^{\prime} H_{2} \leq 2 \varepsilon_{0} \Lambda H_{2}$. Let $\left(t_{0}, X_{0}\right) \in \mathbb{R}^{2 n+1}$ and $\Lambda \geq 1$ such that

$$
Q\left(t_{0}, X_{0}, \Lambda\right)=0 \text { and }\left|Q_{X}^{\prime}\left(t_{0}, X_{0}, \Lambda\right)\right| \geq \frac{1}{2}
$$


The result of the lemma 4.2 .5 implies that

$$
Q_{t}^{\prime}\left(t_{0}, X_{0}, \Lambda\right)>0
$$

Since from (46), $Q \in C^{2[n / 2]+4}\left(\mathbb{R}^{2 n+1}\right)$, we can apply the implicit function theorem to get $\tilde{\theta}_{\Lambda}$ a $C^{2[n / 2]+4}$ function defined on an open neighbourhood $V_{0}$ of $X_{0}$ such that for all $X \in V_{0}$,

$$
Q\left(\tilde{\theta}_{\Lambda}(X), X, \Lambda\right)=0 \text { with } t_{0}=\tilde{\theta}_{\Lambda}\left(X_{0}\right)
$$

Using a Taylor formula at the first order, we deduce from (140) that for all $(t, X) \in \mathbb{R} \times V_{0}$,

$$
\begin{aligned}
Q(t, X, \Lambda) & =Q(t, X, \Lambda)-Q\left(\tilde{\theta}_{\Lambda}(X), X, \Lambda\right) \\
& =\left(\int_{0}^{1} Q_{t}^{\prime}\left((1-s) \tilde{\theta}_{\Lambda}(X)+s t, X, \Lambda\right) d s\right)\left(t-\tilde{\theta}_{\Lambda}(X)\right) .
\end{aligned}
$$

Setting

$$
g(t, X, \Lambda)=\int_{0}^{1} Q_{t}^{\prime}\left((1-s) \tilde{\theta}_{\Lambda}(X)+s t, X, \Lambda\right) d s
$$

we deduce from (138), (139) and (140) that there exists an open neighbourhood $\Omega_{0}$ of the set $\left\{Q=0\right.$ and $\left.\left|Q_{X}^{\prime}\right| \geq 1 / 2\right\}$ such that

$$
\Omega_{0} \subset\left\{\left|Q_{X}^{\prime}\right|>0\right\}
$$

$$
g \in C^{2[n / 2]+3}\left(\Omega_{0}\right), g>0 \text { on } \Omega_{0} \text { and } Q(t, X, \Lambda)=g(t, X, \Lambda)\left(t-\tilde{\theta}_{\Lambda}(X)\right)
$$

on $\Omega_{0}$. It follows from (77) and (145) that for all $(t, X) \in \Omega_{0}$ and $\Lambda \geq 1$,

$$
\tilde{\theta}_{\Lambda}(X)=\theta(X, \Lambda) \text { and } Q(t, X, \Lambda)=g(t, X, \Lambda)(t-\theta(X, \Lambda)) .
$$

This induces that the function $(t, X) \mapsto t-\theta(X, \Lambda)$ is $C^{2[n / 2]+4}$ on $\Omega_{0}$ and that

$$
H(Q(t, X, \Lambda))=H(t-\theta(X, \Lambda))
$$

on $\Omega_{0}$, where $H$ stands for the Heaviside function. By differentiating (146) with respect to $t$, we obtain according to (144) that on $\Omega_{0}$,

$$
Q_{t}^{\prime} \delta(Q)=\delta(t-\theta(X, \Lambda))
$$

TOME $136-2008-\mathrm{N}^{\mathrm{O}} 3$ 
Since from (96) and (97), supp $w_{0}\left(\left|Q_{X}^{\prime}\right|^{2}\right) \subset\left\{\left|Q_{X}^{\prime}\right| \geq 1\right\}$, we deduce from (134), (144) and (147) that

$$
=<\delta(t-\theta(X, \Lambda)) w_{0}\left(\left|Q_{X}^{\prime}\right|^{2}\right) H_{2},|\Phi|^{2}>_{\mathcal{S}^{\prime}, \mathcal{S}}
$$

$$
=\int_{\mathbb{R}^{2 n}} w_{0}\left(\left|Q_{X}^{\prime}(\theta(X, \Lambda), X, \Lambda)\right|^{2}\right) H_{2}(\theta(X, \Lambda), X, \Lambda)|\Phi(\theta(X, \Lambda), X)|^{2} d X
$$

$$
\leq \int_{\mathbb{R}^{2 n}} H_{2}(\theta(X, \Lambda), X, \Lambda)|\Phi(\theta(X, \Lambda), X)|^{2} d X
$$

We obtain from (126), (135) and (148) that

$$
\begin{aligned}
\mid< & {\left[w_{0}\left(\left|Q_{X}^{\prime}\right|^{2}\right) H_{2} \frac{\partial Q}{\partial X} \cdot \frac{\partial \tilde{S}}{\partial X}\right]^{\mathrm{Wick}} u, \bar{u}>_{\mathcal{S}^{\prime}, \mathcal{S}} \mid } \\
& \leq 4 \varepsilon_{0} \sup _{j=1, \ldots, N}\left\|\alpha_{j}^{\prime}\right\|_{L^{\infty}}^{2} \int_{\mathbb{R}^{2 n}} H_{2}(\theta(X, \Lambda), X, \Lambda)|\Phi(\theta(X, \Lambda), X)|^{2} d X
\end{aligned}
$$

Using the triangular inequality, it follows from (54), (98), (108), (110), (116) and (152) that

$$
\begin{aligned}
& \left|<\left[\frac{\partial}{\partial X} \cdot\left(S \frac{\partial Q}{\partial X}\right)\right]^{\mathrm{Wick}} u, \bar{u}>_{\mathcal{S}^{\prime}, \mathcal{S}}\right| \\
\leq & 4 \varepsilon_{0} \sup _{j=1, \ldots, N}\left\|\alpha_{j}^{\prime}\right\|_{L^{\infty}}^{2} \int_{\mathbb{R}^{2 n}} H_{2}(\theta(X, \Lambda), X, \Lambda)|\Phi(\theta(X, \Lambda), X)|^{2} d X \\
& +c_{9}\|u\|_{L^{2}}^{2} \\
\leq & \frac{1}{N} \int_{\mathbb{R}^{2 n}} H_{2}(\theta(X, \Lambda), X, \Lambda)|\Phi(\theta(X, \Lambda), X)|^{2} d X+c_{9}\|u\|_{L^{2}}^{2},
\end{aligned}
$$

where $c_{9}=c_{7}+c_{8}$. Using again the triangular inequality, we obtain from (83) and (90) that

$$
\begin{gathered}
\operatorname{Re}\left(D_{t} u, i S^{\mathrm{Wick}} u\right)_{L^{2}}+\left((S Q)^{\mathrm{Wick}} u, u\right)_{L^{2}} \leq\left|\operatorname{Re}\left(D_{t} u+i Q^{\mathrm{Wick}} u, i S^{\mathrm{Wick}} u\right)_{L^{2}}\right| \\
+\frac{1}{4 \pi}\left|<\left(Q_{X}^{\prime} \cdot S_{X}^{\prime}\right)^{\mathrm{Wick}} u, \bar{u}>_{\mathcal{S}^{\prime}, \mathcal{S}}\right|+\left|\int_{\mathbb{R}}(R(t) u(t, \cdot), u(t, \cdot))_{L^{2}\left(\mathbb{R}^{n}\right)} d t\right| .
\end{gathered}
$$


Using always the triangular inequality, we deduce from (84), (92) and (153) that

$$
\begin{aligned}
\frac{1}{4 \pi}\left|<\left(Q_{X}^{\prime} \cdot S_{X}^{\prime}\right)^{\mathrm{Wick}} u, \bar{u}>_{\mathcal{S}^{\prime}, \mathcal{S}}\right| \leq \frac{1}{4 \pi}\left(2 n \gamma_{1,2}(Q)+c_{9}\right)\|u\|_{L^{2}}^{2} \\
+\frac{1}{4 \pi N} \int_{\mathbb{R}^{2 n}} H_{2}(\theta(X, \Lambda), X, \Lambda)|\Phi(\theta(X, \Lambda), X)|^{2} d X
\end{aligned}
$$

Finally, we obtain with (80), (85), (89), (90), (157) and (158) that

$$
\begin{aligned}
& \frac{1}{2 \pi} \int_{\mathbb{R}^{2 n}} H_{2}(\theta(X, \Lambda), X, \Lambda)|\Phi(\theta(X, \Lambda), X)|^{2} d X-c_{6}\|u\|_{L^{2}}^{2} \\
&+\int_{\mathbb{R}^{2 n+1}} H_{2}(t, X, \Lambda)|Q(t, X, \Lambda) \| \Phi(t, X)|^{2} d t d X \\
& \leq\left\|D_{t} u+i Q^{\mathrm{Wick}} u\right\|_{L^{2}}\|u\|_{L^{2}}+\left(\frac{n}{2 \pi}+d_{n}\right) \gamma_{1,2}(Q)\|u\|_{L^{2}}^{2} \\
&+\frac{1}{4 \pi} c_{9}\|u\|_{L^{2}}^{2}+\frac{1}{4 \pi N} \int_{\mathbb{R}^{2 n}} H_{2}(\theta(X, \Lambda), X, \Lambda)|\Phi(\theta(X, \Lambda), X)|^{2} d X,
\end{aligned}
$$

which induces, since from (6), $N \in \mathbb{N}^{*}$, that there exists a positive constant $c_{10}$ such that for all $u \in C_{0}^{\infty}\left(\mathbb{R}^{n+1}\right)$ and $\Lambda \geq 1$,

$$
\begin{aligned}
& \int_{\mathbb{R}^{2 n+1}} H_{2}(t, X, \Lambda) \mid Q(t, X, \Lambda) \| \Phi(t, X)\left.\right|^{2} d t d X \\
& \leq\left\|D_{t} u+i Q^{\mathrm{Wick}} u\right\|_{L^{2}}\|u\|_{L^{2}}+c_{10}\|u\|_{L^{2}}^{2}
\end{aligned}
$$

This ends the proof of Lemma 4.2.2.

Step 3. In this third step, we estimate the first term of the right-hand-side of (11).

LEMmA 4.2.6. - There exist some positive constants $c_{11}$ and $\Lambda_{0} \geq 1$ such that for all $u \in C_{0}^{\infty}\left(\mathbb{R}^{n+1}\right)$ and $\Lambda \geq \Lambda_{0}$,

$$
\begin{aligned}
& \quad \Lambda^{\frac{1}{N+1}} \int_{\left\{|Q|<\Lambda^{1 /(N+1)}\right\}} H_{2}(t, X, \Lambda)|\Phi(t, X)|^{2} d t d X \leq c_{11}\left\|D_{t} u+i Q^{\text {Wick }} u\right\|_{L^{2}}\|u\|_{L^{2}} \\
& +c_{11}\|u\|_{L^{2}}^{2}+c_{11} \int_{\mathbb{R}^{2 n+1}} H_{2}(t, X, \Lambda)\left|Q(t, X, \Lambda)\left\|\left.\Phi(t, X)\right|^{2} d t d X+\frac{1}{2} \Lambda^{\frac{1}{N+1}}\right\| u \|_{L^{2}}^{2},\right. \\
& \text { if } \Phi(t, X)=W(u(t, \cdot))(X) .
\end{aligned}
$$

Proof of Lemma 4.2.6. - For $\Lambda \geq 1$, let us consider the set

$$
E_{\Lambda}=\left\{(t, X) \in \mathbb{R}^{2 n+1}:|Q(t, X, \Lambda)|<\Lambda^{\frac{1}{N+1}}\right\} .
$$


If $(t, X) \in E_{\Lambda}$, it follows from (45) that

$$
\left|q\left(t, \Lambda^{-\frac{1}{2}} X\right)\right|<\Lambda^{-\frac{N}{N+1}} \text {. }
$$

Since from (6), $N \in \mathbb{N}^{*}$, we deduce from (5) and (161) that there exists a constant $\Lambda_{0} \geq 1$ such that for all $\Lambda \geq \Lambda_{0}$,

$$
E_{\Lambda} \subset[-A, A] \times \mathbb{R}^{2 n}
$$

Then, using (6), (7), (161) and (162), we obtain that if $(t, X) \in E_{\Lambda}$ and $\Lambda \geq \Lambda_{0}$,

$$
M_{0} \prod_{j=1}^{N}\left|t-\alpha_{j}\left(\Lambda^{-\frac{1}{2}} X\right)\right| \leq e\left(t, \Lambda^{-\frac{1}{2}} X\right) \prod_{j=1}^{N}\left|t-\alpha_{j}\left(\Lambda^{-\frac{1}{2}} X\right)\right|<\Lambda^{-\frac{N}{N+1}},
$$

which implies that there exists $j_{0} \in\{1, \ldots, N\}$ such that

$$
M_{0}^{\frac{1}{N}}\left|t-\alpha_{j_{0}}\left(\Lambda^{-\frac{1}{2}} X\right)\right|<\Lambda^{-\frac{1}{N+1}} .
$$

Let us consider $\chi_{3}$ a $C_{0}^{\infty}(\mathbb{R},[0,1])$ function such that

$$
\text { supp } \chi_{3} \subset[-2,2] \text { and } \chi_{3}=1 \text { on }[-1,1] \text {. }
$$

We deduce from (163) and (164) that for all $(t, X) \in E_{\Lambda}$ and $\Lambda \geq \Lambda_{0}$,

$$
1 \leq \sum_{j=1}^{N} \chi_{3}\left(M_{0}^{\frac{1}{N}} \Lambda^{\frac{1}{N+1}}\left(t-\alpha_{j}\left(\Lambda^{-\frac{1}{2}} X\right)\right)\right)
$$

which implies according to (160) that

$$
\begin{aligned}
& \text { (165) } \Lambda^{\frac{1}{N+1}} \int_{\left\{|Q|<\Lambda^{1 /(N+1)}\right\}} H_{2}(t, X, \Lambda)|\Phi(t, X)|^{2} d t d X \\
& \leq \sum_{j=1}^{N} \int_{\mathbb{R}^{2 n+1}} \Lambda^{\frac{1}{N+1}} H_{2}(t, X, \Lambda) \chi_{3}\left(M_{0}^{\frac{1}{N}} \Lambda^{\frac{1}{N+1}}\left(t-\alpha_{j}\left(\Lambda^{-\frac{1}{2}} X\right)\right)\right)|\Phi(t, X)|^{2} d t d X .
\end{aligned}
$$

Let $j_{0} \in\{1, \ldots, N\}$, we define the following multiplier

$$
\text { (166) } m(t, X, \Lambda)=H_{2}(t, X, \Lambda) \int_{-\infty}^{t} \Lambda^{\frac{1}{N+1}} \chi_{3}\left(M_{0}^{\frac{1}{N}} \Lambda^{\frac{1}{N+1}}\left(s-\alpha_{j_{0}}\left(\Lambda^{-\frac{1}{2}} X\right)\right)\right) d s .
$$

Using a change of variables, we first notice from (134), (164) and (166) that for all $(t, X) \in \mathbb{R}^{2 n+1}$ and $\Lambda \geq 1$,

$$
\begin{aligned}
& |m(t, X, \Lambda)| \leq M_{0}^{-\frac{1}{N}} \int_{-\infty}^{M_{0}^{\frac{1}{N}} \Lambda^{\frac{1}{N+1}}\left(t-\alpha_{j_{0}}\left(\Lambda^{-\frac{1}{2}} X\right)\right)} \begin{array}{c}
\chi_{3}(u) d u \\
\gamma^{2}
\end{array} \\
& \leq M_{0}^{-\frac{1}{N}} \int_{\mathbb{R}} \chi_{3}(u) d u<+\infty,
\end{aligned}
$$

i.e.,

$$
\|m\|_{L^{\infty}\left(\mathbb{R}^{2 n+1}\right)} \leq M_{0}^{-\frac{1}{N}}\left\|\chi_{3}\right\|_{L^{1}(\mathbb{R})}<+\infty .
$$


One has from (166) that

$$
\begin{array}{r}
\partial_{t} m(t, X, \Lambda)=H_{2}(t, X, \Lambda) \Lambda^{\frac{1}{N+1}} \chi_{3}\left(M_{0}^{\frac{1}{N}} \Lambda^{\frac{1}{N+1}}\left(t-\alpha_{j_{0}}\left(\Lambda^{-\frac{1}{2}} X\right)\right)\right) \\
+A(t, X, \Lambda)
\end{array}
$$

where

$$
A(t, X, \Lambda)=\partial_{t} H_{2}(t, X, \Lambda) \int_{-\infty}^{t} \Lambda^{\frac{1}{N+1}} \chi_{3}\left(M_{0}^{\frac{1}{N}} \Lambda^{\frac{1}{N+1}}\left(s-\alpha_{j_{0}}\left(\Lambda^{-\frac{1}{2}} X\right)\right)\right) d s .
$$

Since from (45) and (55), a direct computation gives

$$
\begin{aligned}
& \left|\partial_{t} H_{2}(t, X, \Lambda)\right|=\left|\chi_{2}^{\prime}\left(Q_{t}^{\prime}(t, X, \Lambda) \varepsilon_{0}^{-1} \Lambda^{-1}\right) Q_{t t}^{\prime \prime}(t, X, \Lambda) \varepsilon_{0}^{-1} \Lambda^{-1}\right| \\
= & \left|\chi_{2}^{\prime}\left(Q_{t}^{\prime}(t, X, \Lambda) \varepsilon_{0}^{-1} \Lambda^{-1}\right) q_{t t}^{\prime \prime}\left(t, \Lambda^{-\frac{1}{2}} X\right) \varepsilon_{0}^{-1}\right| \leq \varepsilon_{0}^{-1}\left\|\chi_{2}^{\prime}\right\|_{L^{\infty}(\mathbb{R})}\left\|q_{t t}^{\prime \prime}\right\|_{L^{\infty}\left(\mathbb{R}^{2 n+1}\right)},
\end{aligned}
$$

we deduce using the same change of variables as in (167) from (3) and (53) that there exists a positive constant $c_{12}$ such that for all $\Lambda \geq 1$,

$$
\|A\|_{L^{\infty}\left(\mathbb{R}^{2 n+1}\right)} \leq \varepsilon_{0}^{-1} M_{0}^{-\frac{1}{N}}\left\|\chi_{3}\right\|_{L^{1}(\mathbb{R})}\left\|\chi_{2}^{\prime}\right\|_{L^{\infty}(\mathbb{R})}\left\|q_{t t}^{\prime \prime}\right\|_{L^{\infty}\left(\mathbb{R}^{2 n+1}\right)} \leq c_{12} .
$$

We also need to make some estimates on the gradient of the multiplier $m(t, X, \Lambda)$ with respect to $X$. We get from (166) that

$$
m_{X}^{\prime}(t, X, \Lambda)=B(t, X, \Lambda)+C(t, X, \Lambda),
$$

where

$$
B(t, X, \Lambda)=\nabla_{X} H_{2}(t, X, \Lambda) \int_{-\infty}^{t} \Lambda^{\frac{1}{N+1}} \chi_{3}\left(M_{0}^{\frac{1}{N}} \Lambda^{\frac{1}{N+1}}\left(s-\alpha_{j_{0}}\left(\Lambda^{-\frac{1}{2}} X\right)\right)\right) d s
$$

and

$$
\begin{aligned}
C(t, X, \Lambda)= & -H_{2}(t, X, \Lambda) \alpha_{j_{0}}^{\prime}\left(\Lambda^{-\frac{1}{2}} X\right) \\
& \times \int_{-\infty}^{t} \Lambda^{\frac{2}{N+1}-\frac{1}{2}} M_{0}^{\frac{1}{N}} \chi_{3}^{\prime}\left(M_{0}^{\frac{1}{N}} \Lambda^{\frac{1}{N+1}}\left(s-\alpha_{j_{0}}\left(\Lambda^{-\frac{1}{2}} X\right)\right)\right) d s .
\end{aligned}
$$

According to (164), we can compute the integral in (175) to obtain that

$$
\begin{aligned}
C(t, X, \Lambda)=-H_{2}(t, X, \Lambda) \alpha_{j_{0}}^{\prime}\left(\Lambda^{-\frac{1}{2}} X\right) \Lambda^{\frac{1}{N+1}-\frac{1}{2}} & \\
& \times \chi_{3}\left(M_{0}^{\frac{1}{N}} \Lambda^{\frac{1}{N+1}}\left(t-\alpha_{j_{0}}\left(\Lambda^{-\frac{1}{2}} X\right)\right)\right),
\end{aligned}
$$

because

$$
\begin{aligned}
& \frac{d}{d s}\left[\chi_{3}\left(M_{0}^{\frac{1}{N}} \Lambda^{\frac{1}{N+1}}\left(s-\alpha_{j_{0}}\left(\Lambda^{-\frac{1}{2}} X\right)\right)\right)\right]=M_{0}^{\frac{1}{N}} \Lambda^{\frac{1}{N+1}} \chi_{3}^{\prime}\left(M_{0}^{\frac{1}{N}} \Lambda^{\frac{1}{N+1}}\left(s-\alpha_{j_{0}}\left(\Lambda^{-\frac{1}{2}} X\right)\right)\right) . \\
& \quad \text { томе } 136-2008-\mathrm{N}^{\mathrm{o}} 3
\end{aligned}
$$


Using (46), (56), (174) and the same change of variables as in (167), we get that for all $\Lambda \geq 1$,

$$
\begin{aligned}
\left\|B \cdot Q_{X}^{\prime}\right\|_{L^{\infty}\left(\mathbb{R}^{2 n+1}\right)} & \leq \gamma_{0,1}\left(H_{2}\right) \Lambda^{-\frac{1}{2}} M_{0}^{-\frac{1}{N}}\left\|\chi_{3}\right\|_{L^{1}(\mathbb{R})} \Lambda^{\frac{1}{2}} \gamma_{1,1}(Q) \\
& \leq \gamma_{0,1}\left(H_{2}\right) \gamma_{1,1}(Q) M_{0}^{-\frac{1}{N}}\left\|\chi_{3}\right\|_{L^{1}(\mathbb{R})}<+\infty .
\end{aligned}
$$

To estimate the term $\left\|C \cdot Q_{X}^{\prime}\right\|_{L^{\infty}\left(\mathbb{R}^{2 n+1}\right)}$, we start by using the Taylor formula at the first order to write that

$$
\begin{aligned}
& Q_{X}^{\prime}(t, X, \Lambda)=Q_{X}^{\prime}\left(\alpha_{j_{0}}\left(\Lambda^{-\frac{1}{2}} X\right), X, \Lambda\right) \\
& \quad+\left(\int_{0}^{1} Q_{X, t}^{\prime \prime}\left((1-s) \alpha_{j_{0}}\left(\Lambda^{-\frac{1}{2}} X\right)+s t, X, \Lambda\right) d s\right)\left(t-\alpha_{j_{0}}\left(\Lambda^{-\frac{1}{2}} X\right)\right) .
\end{aligned}
$$

Since from (3) and (45),

$$
Q_{t}^{\prime} \in S_{2[n / 2]+3}\left(\Lambda, \Lambda^{-1} d X^{2}\right)
$$

uniformly with respect to the parameter $t$ in $\mathbb{R}$, we deduce using the triangular inequality and (179) that for all $(t, X) \in \mathbb{R}^{2 n+1}$ and $\Lambda \geq 1$,

$$
\left|Q_{X}^{\prime}(t, X, \Lambda)\right| \leq\left|Q_{X}^{\prime}\left(\alpha_{j_{0}}\left(\Lambda^{-\frac{1}{2}} X\right), X, \Lambda\right)\right|+\gamma_{1,1}\left(Q_{t}^{\prime}\right) \Lambda^{\frac{1}{2}}\left|t-\alpha_{j_{0}}\left(\Lambda^{-\frac{1}{2}} X\right)\right|
$$

Then, we obtain using (176), (181) and the triangular inequality that for all $(t, X) \in \mathbb{R}^{2 n+1}$ and $\Lambda \geq 1$,

$$
\left|C(t, X, \Lambda) \cdot Q_{X}^{\prime}(t, X, \Lambda)\right| \leq D(t, X, \Lambda)+F(t, X, \Lambda),
$$

where

$$
\begin{aligned}
D(t, X, \Lambda) & =\Lambda^{\frac{1}{N+1}-\frac{1}{2}}\left\|\alpha_{j_{0}}^{\prime}\right\|_{L^{\infty}\left(\mathbb{R}^{2 n}\right)} H_{2}(t, X, \Lambda) \\
& \times \chi_{3}\left(M_{0}^{\frac{1}{N}} \Lambda^{\frac{1}{N+1}}\left(t-\alpha_{j_{0}}\left(\Lambda^{-\frac{1}{2}} X\right)\right)\right)\left|Q_{X}^{\prime}\left(\alpha_{j_{0}}\left(\Lambda^{-\frac{1}{2}} X\right), X, \Lambda\right)\right|
\end{aligned}
$$

and

$$
\begin{aligned}
F(t, X, \Lambda) & =\Lambda^{\frac{1}{N+1}}\left\|\alpha_{j_{0}}^{\prime}\right\|_{L^{\infty}\left(\mathbb{R}^{2 n}\right)} H_{2}(t, X, \Lambda) \\
\times & \chi_{3}\left(M_{0}^{\frac{1}{N}} \Lambda^{\frac{1}{N+1}}\left(t-\alpha_{j_{0}}\left(\Lambda^{-\frac{1}{2}} X\right)\right)\right) \gamma_{1,1}\left(Q_{t}^{\prime}\right)\left|t-\alpha_{j_{0}}\left(\Lambda^{-\frac{1}{2}} X\right)\right|
\end{aligned}
$$

Since from (164),

$$
\begin{aligned}
\Lambda^{\frac{1}{N+1}}\left|t-\alpha_{j_{0}}\left(\Lambda^{-\frac{1}{2}} X\right)\right| \chi_{3}\left(M_{0}^{\frac{1}{N}} \Lambda^{\frac{1}{N+1}}\left(t-\alpha_{j_{0}}\left(\Lambda^{-\frac{1}{2}} X\right)\right)\right) & \\
& \leq\left\|x \chi_{3}(x)\right\|_{L^{\infty}(\mathbb{R})} M_{0}^{-\frac{1}{N}}<+\infty
\end{aligned}
$$

we deduce from (134) and (184) that there exists a positive constant $c_{13}$ such that for all $\Lambda \geq 1$,

$$
\|F\|_{L^{\infty}\left(\mathbb{R}^{2 n+1}\right)} \leq\left\|\alpha_{j_{0}}^{\prime}\right\|_{L^{\infty}\left(\mathbb{R}^{2 n}\right)}\left\|x \chi_{3}(x)\right\|_{L^{\infty}(\mathbb{R})} M_{0}^{-\frac{1}{N}} \gamma_{1,1}\left(Q_{t}^{\prime}\right) \leq c_{13} .
$$


To get an upper bound on the $L^{\infty}$-norm of $D$, we first notice from (132) that we have

$$
Q_{t}^{\prime}\left(\alpha_{j_{0}}\left(\Lambda^{-\frac{1}{2}} X\right), X, \Lambda\right) \alpha_{j_{0}}^{\prime}\left(\Lambda^{-\frac{1}{2}} X\right) \Lambda^{-\frac{1}{2}}+Q_{X}^{\prime}\left(\alpha_{j_{0}}\left(\Lambda^{-\frac{1}{2}} X\right), X, \Lambda\right)=0,
$$

for a.e. $X$ in $\mathbb{R}^{2 n}$. It follows from (56), (183) and (186) that for all $\Lambda \geq 1$,

$$
\|D\|_{L^{\infty}\left(\mathbb{R}^{2 n+1}\right)} \leq \Lambda^{\frac{1}{N+1}-1}\left\|\alpha_{j_{0}}^{\prime}\right\|_{L^{\infty}\left(\mathbb{R}^{2 n}\right)}^{2}
$$

$\times\left\|H_{2}(t, X, \Lambda) \chi_{3}\left(M_{0}^{\frac{1}{N}} \Lambda^{\frac{1}{N+1}}\left(t-\alpha_{j_{0}}\left(\Lambda^{-\frac{1}{2}} X\right)\right)\right) Q_{t}^{\prime}\left(\alpha_{j_{0}}\left(\Lambda^{-\frac{1}{2}} X\right), X, \Lambda\right)\right\|_{L^{\infty}\left(\mathbb{R}^{2 n+1}\right)}$.

Since from (3) and (45),

$$
Q_{t t}^{\prime \prime} \in S_{2[n / 2]+2}\left(\Lambda, \Lambda^{-1} d X^{2}\right),
$$

uniformly with respect to the parameter $t$ in $\mathbb{R}$, we can use that

$$
\begin{aligned}
& Q_{t}^{\prime}\left(\alpha_{j_{0}}\left(\Lambda^{-\frac{1}{2}} X\right), X, \Lambda\right)=Q_{t}^{\prime}(t, X, \Lambda) \\
& \quad+\left(\int_{0}^{1} Q_{t t}^{\prime \prime}\left((1-s) t+s \alpha_{j_{0}}\left(\Lambda^{-\frac{1}{2}} X\right), X, \Lambda\right) d s\right)\left(\alpha_{j_{0}}\left(\Lambda^{-\frac{1}{2}} X\right)-t\right)
\end{aligned}
$$

to obtain that for all $(t, X) \in \mathbb{R}^{2 n+1}$ and $\Lambda \geq 1$,

$$
Q_{t}^{\prime}\left(\alpha_{j_{0}}\left(\Lambda^{-\frac{1}{2}} X\right), X, \Lambda\right) \leq Q_{t}^{\prime}(t, X, \Lambda)+\gamma_{1,0}\left(Q_{t t}^{\prime \prime}\right) \Lambda\left|t-\alpha_{j_{0}}\left(\Lambda^{-\frac{1}{2}} X\right)\right| .
$$

Now, we get from (76) that for all $X \in \mathbb{R}^{2 n}$ and $\Lambda \geq 1$,

$$
Q_{t}^{\prime}\left(\alpha_{j_{0}}\left(\Lambda^{-\frac{1}{2}} X\right), X, \Lambda\right) \geq 0,
$$

because, if there exist $X_{0} \in \mathbb{R}^{2 n}$ and $\tilde{\Lambda}_{0} \geq 1$ such that $Q_{t}^{\prime}\left(\alpha_{j_{0}}\left(\tilde{\Lambda}_{0}^{-\frac{1}{2}} X_{0}\right), X_{0}, \tilde{\Lambda}_{0}\right)$ $<0$, we will obtain from (131) that the function

$$
t \mapsto Q\left(t, X_{0}, \tilde{\Lambda}_{0}\right),
$$

changes sign from positive values to negative ones at the first order in $t=$ $\alpha_{j_{0}}\left(\tilde{\Lambda}_{0}^{-\frac{1}{2}} X_{0}\right)$, which is not allowed according to (76). It follows from (134), (164), (188) and (189) that for all $(t, X) \in \mathbb{R}^{2 n+1}$ and $\Lambda \geq 1$,

$$
\left|H_{2}(t, X, \Lambda) \chi_{3}\left(M_{0}^{\frac{1}{N}} \Lambda^{\frac{1}{N+1}}\left(t-\alpha_{j_{0}}\left(\Lambda^{-\frac{1}{2}} X\right)\right)\right) Q_{t}^{\prime}\left(\alpha_{j_{0}}\left(\Lambda^{-\frac{1}{2}} X\right), X, \Lambda\right)\right|
$$

$$
=H_{2}(t, X, \Lambda) \chi_{3}\left(M_{0}^{\frac{1}{N}} \Lambda^{\frac{1}{N+1}}\left(t-\alpha_{j_{0}}\left(\Lambda^{-\frac{1}{2}} X\right)\right)\right) Q_{t}^{\prime}\left(\alpha_{j_{0}}\left(\Lambda^{-\frac{1}{2}} X\right), X, \Lambda\right)
$$

$$
\leq H_{2}(t, X, \Lambda) Q_{t}^{\prime}(t, X, \Lambda)
$$

$$
+\chi_{3}\left(M_{0}^{\frac{1}{N}} \Lambda^{\frac{1}{N+1}}\left(t-\alpha_{j_{0}}\left(\Lambda^{-\frac{1}{2}} X\right)\right)\right) \gamma_{1,0}\left(Q_{t t}^{\prime \prime}\right) \Lambda\left|t-\alpha_{j_{0}}\left(\Lambda^{-\frac{1}{2}} X\right)\right|,
$$


because $0 \leq H_{2} \leq 1$ and $0 \leq \chi_{3} \leq 1$. Since from (57) and (134), one has for all $(t, X) \in \mathbb{R}^{2 n+1}$ and $\Lambda \geq 1$,

$$
H_{2}(t, X, \Lambda) Q_{t}^{\prime}(t, X, \Lambda) \leq 2 \varepsilon_{0} \Lambda H_{2}(t, X, \Lambda) \leq 2 \varepsilon_{0} \Lambda,
$$

and that

$\chi_{3}\left(M_{0}^{\frac{1}{N}} \Lambda^{\frac{1}{N+1}}\left(t-\alpha_{j_{0}}\left(\Lambda^{-\frac{1}{2}} X\right)\right)\right)\left|t-\alpha_{j_{0}}\left(\Lambda^{-\frac{1}{2}} X\right)\right| \leq \Lambda^{-\frac{1}{N+1}} M_{0}^{-\frac{1}{N}}\left\|x \chi_{3}(x)\right\|_{L^{\infty}(\mathbb{R})}$, we deduce from (164), (187) and (190) that there exists a positive constant $c_{14}$ such that for all $\Lambda \geq 1$,

$\|D\|_{L^{\infty}\left(\mathbb{R}^{2 n+1}\right)} \leq \Lambda^{\frac{1}{N+1}-1}\left\|\alpha_{j_{0}}^{\prime}\right\|_{L^{\infty}}^{2}\left(2 \varepsilon_{0} \Lambda+\Lambda^{1-\frac{1}{N+1}} \gamma_{1,0}\left(Q_{t t}^{\prime \prime}\right) M_{0}^{-\frac{1}{N}}\left\|x \chi_{3}(x)\right\|_{L^{\infty}(\mathbb{R})}\right)$

$$
\leq 2 \varepsilon_{0} \Lambda^{\frac{1}{N+1}}\left\|\alpha_{j_{0}}^{\prime}\right\|_{L^{\infty}}^{2}+c_{14} .
$$

It follows from (182), (185) and (194) that for all $\Lambda \geq 1$,

$$
\left\|C \cdot Q_{X}^{\prime}\right\|_{L^{\infty}\left(\mathbb{R}^{2 n+1}\right)} \leq 2 \varepsilon_{0}\left\|\alpha_{j_{0}}^{\prime}\right\|_{L^{\infty}}^{2} \Lambda^{\frac{1}{N+1}}+c_{13}+c_{14} .
$$

Using the triangular inequality, we finally obtain from (54), (173), (177) and (196) that there exists a positive constant $c_{15}$ such that for all $\Lambda \geq 1$,

$$
\begin{aligned}
\left\|m_{X}^{\prime} \cdot Q_{X}^{\prime}\right\|_{L^{\infty}\left(\mathbb{R}^{2 n+1}\right)} & \leq 2 \varepsilon_{0}\left\|\alpha_{j_{0}}^{\prime}\right\|_{L^{\infty}}^{2} \Lambda^{\frac{1}{N+1}}+c_{15} \\
& \leq \frac{1}{2 N} \Lambda^{\frac{1}{N+1}}+c_{15} .
\end{aligned}
$$

We can now use our multiplier $m^{\text {Wick }}$ to get that

$$
2 \operatorname{Re}\left(D_{t} u+i Q^{\mathrm{Wick}} u, i m^{\mathrm{Wick}} u\right)_{L^{2}}=2 \operatorname{Re}\left(D_{t} u, i m^{\mathrm{Wick}} u\right)_{L^{2}}
$$$$
+2 \operatorname{Re}\left(Q^{\text {Wick }} u, m^{\text {Wick }} u\right)_{L^{2}}=\left(\left[D_{t}, i m^{\text {Wick }}\right] u, u\right)_{L^{2}}+2\left(\operatorname{Re}\left(m^{\text {Wick }} Q^{\text {Wick }}\right) u, u\right)_{L^{2}},
$$

because, since $m$ is a real-valued function, the operators $m^{\text {Wick }}$ and $i m^{\text {Wick }}$ are respectively some self-adjoint and anti-self-adjoint operators. Using the Cauchy-Schwarz inequality, we first obtain from Proposition 3.2.1 and (169) that there exists a positive constant $c_{16}$ such that for all $u \in C_{0}^{\infty}\left(\mathbb{R}^{n+1}\right)$ and $\Lambda \geq 1$,

$$
\begin{aligned}
& 2\left|\operatorname{Re}\left(D_{t} u+i Q^{\mathrm{Wick}} u, i m^{\text {Wick }} u\right)_{L^{2}}\right| \leq 2\left\|D_{t} u+i Q^{\text {Wick }} u\right\|_{L^{2}}\left\|m^{\text {Wick }} u\right\|_{L^{2}} \\
& \leq 2\|m\|_{L^{\infty}}\left\|D_{t} u+i Q^{\text {Wick }} u\right\|_{L^{2}}\|u\|_{L^{2}} \leq c_{16}\left\|D_{t} u+i Q^{\text {Wick }} u\right\|_{L^{2}}\|u\|_{L^{2}} .
\end{aligned}
$$

Then, since from Proposition 3.2.1, one has

$$
\begin{gathered}
\left(\left[D_{t}, i m^{\text {Wick }}\right] u, u\right)_{L^{2}}=\frac{1}{2 \pi}\left(\left(\partial_{t} m\right)^{\text {Wick }} u, u\right)_{L^{2}}=\frac{1}{2 \pi}\left(W^{*}\left(\partial_{t} m\right) W u, u\right)_{L^{2}\left(\mathbb{R}^{n+1}\right)} \\
\quad=\frac{1}{2 \pi}\left(\partial_{t} m W u, W u\right)_{L^{2}\left(\mathbb{R}^{2 n+1}\right)}=\frac{1}{2 \pi} \int_{\mathbb{R}^{2 n+1}} \partial_{t} m(t, X, \Lambda)|\Phi(t, X)|^{2} d t d X
\end{gathered}
$$


if $\Phi(t, X)=W(u(t, \cdot))(X)$, we deduce from (170) that

$$
\left(\left[D_{t}, i m^{\mathrm{Wick}}\right] u, u\right)_{L^{2}}=\frac{1}{2 \pi} \int_{\mathbb{R}^{2 n+1}} A(t, X, \Lambda)|\Phi(t, X)|^{2} d t d X
$$

$+\frac{1}{2 \pi} \int_{\mathbb{R}^{2 n+1}} \Lambda^{\frac{1}{N+1}} H_{2}(t, X, \Lambda) \chi_{3}\left(M_{0}^{\frac{1}{N}} \Lambda^{\frac{1}{N+1}}\left(t-\alpha_{j_{0}}\left(\Lambda^{-\frac{1}{2}} X\right)\right)\right)|\Phi(t, X)|^{2} d t d X$.

Since from (172),

$$
\begin{aligned}
\left.\left|\frac{1}{2 \pi} \int_{\mathbb{R}^{2 n+1}} A(t, X, \Lambda)\right| \Phi(t, X)\right|^{2} d t d X \mid & \leq \frac{1}{2 \pi}\|A\|_{L^{\infty}} \int_{\mathbb{R}^{2 n+1}}|\Phi(t, X)|^{2} d t d X \\
& \leq \frac{c_{12}}{2 \pi}\|W u\|_{L^{2}\left(\mathbb{R}^{2 n+1}\right)}^{2}=\frac{c_{12}}{2 \pi}\|u\|_{L^{2}\left(\mathbb{R}^{n+1}\right)}^{2},
\end{aligned}
$$

because $W$ is an isometric mapping from $L^{2}\left(\mathbb{R}_{x}^{n}\right)$ to $L^{2}\left(\mathbb{R}_{X}^{2 n}\right)$, we obtain from (201) that for all $u \in C_{0}^{\infty}\left(\mathbb{R}^{n+1}\right)$ and $\Lambda \geq 1$,

(202) $\left(\left[D_{t}, i m^{\mathrm{Wick}}\right] u, u\right)_{L^{2}} \geq$

$$
\begin{array}{r}
\frac{1}{2 \pi} \int_{\mathbb{R}^{2 n+1}} \Lambda^{\frac{1}{N+1}} H_{2}(t, X, \Lambda) \chi_{3}\left(M_{0}^{\frac{1}{N}} \Lambda^{\frac{1}{N+1}}\left(t-\alpha_{j_{0}}\left(\Lambda^{-\frac{1}{2}} X\right)\right)\right)|\Phi(t, X)|^{2} d t d X \\
-\frac{c_{12}}{2 \pi}\|u\|_{L^{2}}^{2} .
\end{array}
$$

Now, according to (46) and (169), we can apply the proposition 3.2.2 to obtain that

$$
\begin{aligned}
\left(\operatorname{Re}\left(m^{\mathrm{Wick}} Q^{\mathrm{Wick}}\right) u, u\right)_{L^{2}}=\left((m Q)^{\mathrm{Wick}} u\right. & , u)_{L^{2}}-\frac{1}{4 \pi}\left(\left(m_{X}^{\prime} \cdot Q_{X}^{\prime}\right)^{\mathrm{Wick}} u, u\right)_{L^{2}} \\
& +\int_{\mathbb{R}}(R(t) u(t, \cdot), u(t, \cdot))_{L^{2}\left(\mathbb{R}^{n}\right)} d t
\end{aligned}
$$

where for all $t \in \mathbb{R}$,

$$
\|R(t)\|_{\mathcal{L}\left(L^{2}\left(\mathbb{R}^{n}\right)\right)} \leq d_{n} M_{0}^{-\frac{1}{N}}\left\|\chi_{3}\right\|_{L^{1}(\mathbb{R})} \gamma_{1,2}(Q)<+\infty .
$$

We deduce from the Cauchy-Schwarz inequality, Proposition 3.2.1 and (197) that

$$
\begin{aligned}
\left|\left(\left(m_{X}^{\prime} \cdot Q_{X}^{\prime}\right)^{\mathrm{Wick}} u, u\right)_{L^{2}}\right| & \leq\left\|\left(m_{X}^{\prime} \cdot Q_{X}^{\prime}\right)^{\mathrm{Wick}} u\right\|_{L^{2}}\|u\|_{L^{2}} \\
& \leq\left\|\left(m_{X}^{\prime} \cdot Q_{X}^{\prime}\right)^{\mathrm{Wick}}\right\|_{\mathcal{L}\left(L^{2}\right)}\|u\|_{L^{2}}^{2} \\
& \leq\left\|m_{X}^{\prime} \cdot Q_{X}^{\prime}\right\|_{L^{\infty}}\|u\|_{L^{2}}^{2} \\
& \leq\left(\frac{1}{2 N} \Lambda^{\frac{1}{N+1}}+c_{15}\right)\|u\|_{L^{2}}^{2} .
\end{aligned}
$$


We also deduce from (204) that there exists a positive constant $c_{17}$ such that for all $u \in C_{0}^{\infty}\left(\mathbb{R}^{n+1}\right)$ and $\Lambda \geq 1$,

$$
\begin{aligned}
\left|\int_{\mathbb{R}}(R(t) u(t, \cdot), u(t, \cdot))_{L^{2}\left(\mathbb{R}^{n}\right)} d t\right| & \leq \int_{\mathbb{R}}\|R(t)\|_{\mathcal{L}\left(L^{2}\left(\mathbb{R}^{n}\right)\right)}\|u(t, \cdot)\|_{L^{2}\left(\mathbb{R}^{n}\right)}^{2} d t \\
& \leq c_{17}\|u\|_{L^{2}}^{2} .
\end{aligned}
$$

Since from Proposition 3.2.1, (134), (166) and (167),

$$
\begin{aligned}
& \left|\left((m Q)^{\mathrm{Wick}} u, u\right)_{L^{2}}\right|=\left|\left(W^{*}(m Q) W u, u\right)_{L^{2}}\right| \\
= & \left|(m Q W u, W u)_{L^{2}}\right|=\left.\left|\int_{\mathbb{R}^{2 n+1}} m Q\right| \Phi\right|^{2} d t d X \mid \\
\leq & \int_{\mathbb{R}^{2 n+1}} H_{2}(t, X, \Lambda)|Q(t, X, \Lambda)| M_{0}^{-\frac{1}{N}}\left\|\chi_{3}\right\|_{L^{1}(\mathbb{R})}|\Phi(t, X)|^{2} d t d X,
\end{aligned}
$$

if $\Phi(t, X)=W(u(t, \cdot))(X)$, we deduce using the triangular inequality, (203), (205), (209) and (211) that there exists a positive constant $c_{18}$ such that for all $u \in C_{0}^{\infty}\left(\mathbb{R}^{n+1}\right)$ and $\Lambda \geq 1$,

$$
\begin{aligned}
\left|\left(\operatorname{Re}\left(m^{\text {Wick }} Q^{\text {Wick }}\right) u, u\right)_{L^{2}}\right| \leq c_{18}\|u\|_{L^{2}}^{2}+\frac{1}{8 \pi N} \Lambda^{\frac{1}{N+1}}\|u\|_{L^{2}}^{2} \\
\quad+c_{18} \int_{\mathbb{R}^{2 n+1}} H_{2}(t, X, \Lambda)|Q(t, X, \Lambda) \| \Phi(t, X)|^{2} d t d X .
\end{aligned}
$$

Then, using again the triangular inequality, it follows (199), (200), (202) and (214) that there exists a positive constant $c_{19}$ such that for all $u \in C_{0}^{\infty}\left(\mathbb{R}^{n+1}\right)$ and $\Lambda \geq 1$,

$$
\frac{1}{2 \pi} \int_{\mathbb{R}^{2 n+1}} \Lambda^{\frac{1}{N+1}} H_{2}(t, X, \Lambda) \chi_{3}\left(M_{0}^{\frac{1}{N}} \Lambda^{\frac{1}{N+1}}\left(t-\alpha_{j_{0}}\left(\Lambda^{-\frac{1}{2}} X\right)\right)\right)|\Phi(t, X)|^{2} d t d X
$$

$$
\leq c_{19}\left\|D_{t} u+i Q^{\mathrm{Wick}} u\right\|_{L^{2}}\|u\|_{L^{2}}+c_{19}\|u\|_{L^{2}}^{2}+\frac{1}{4 \pi N} \Lambda^{\frac{1}{N+1}}\|u\|_{L^{2}}^{2}
$$

$$
+c_{19} \int_{\mathbb{R}^{2 n+1}} H_{2}(t, X, \Lambda)|Q(t, X, \Lambda)||\Phi(t, X)|^{2} d t d X .
$$

We finally deduce from (165) and (215) that there exists a positive constant $c_{20}$ such that for all $u \in C_{0}^{\infty}\left(\mathbb{R}^{n+1}\right)$ and $\Lambda \geq \Lambda_{0}$,

$$
\begin{gathered}
\Lambda^{\frac{1}{N+1}} \int_{\left\{|Q|<\Lambda^{1 /(N+1)}\right\}} H_{2}(t, X, \Lambda)|\Phi(t, X)|^{2} d t d X \leq c_{20}\left\|D_{t} u+i Q^{\mathrm{Wick}} u\right\|_{L^{2}}\|u\|_{L^{2}} \\
+c_{20}\|u\|_{L^{2}}^{2}+\frac{1}{2} \Lambda^{\frac{1}{N+1}}\|u\|_{L^{2}}^{2}+c_{20} \int_{\mathbb{R}^{2 n+1}} H_{2}(t, X, \Lambda)|Q(t, X, \Lambda) \| \Phi(t, X)|^{2} d t d X,
\end{gathered}
$$


which ends the proof of Lemma 4.2.6.

Last step. Using the first three steps, we can now prove the estimate (44). We first use the phase space cut-off (56) to obtain using Proposition 3.2.1 and Lemma 4.2.1 that for all $u \in C_{0}^{\infty}\left(\mathbb{R}^{n+1}\right)$ and $\Lambda \geq 1$,

$$
\begin{aligned}
& \text { (218) } \Lambda^{\frac{1}{N+1}}\|u\|_{L^{2}}^{2}=\Lambda^{\frac{1}{N+1}}\left(H_{1}^{\mathrm{Wick}} u, u\right)_{L^{2}}+\Lambda^{\frac{1}{N+1}}\left(H_{2}^{\mathrm{Wick}} u, u\right)_{L^{2}} \\
& \leq c_{1}^{-1} \Lambda^{-\frac{N}{N+1}}\left\|D_{t} u+i Q^{\mathrm{Wick}} u\right\|_{L^{2}}^{2}+c_{1}^{-1} \Lambda^{-\frac{N}{N+1}}\|u\|_{L^{2}}^{2}+\Lambda^{\frac{1}{N+1}}\left(H_{2}^{\text {Wick }} u, u\right)_{L^{2}} .
\end{aligned}
$$

Using Proposition 3.2.1, we get that

$$
\begin{aligned}
\Lambda^{\frac{1}{N+1}}\left(H_{2}^{\text {Wick }} u, u\right)_{L^{2}\left(\mathbb{R}^{n+1}\right)} & =\Lambda^{\frac{1}{N+1}}\left(W^{*} H_{2} W u, u\right)_{L^{2}\left(\mathbb{R}^{n+1}\right)} \\
=\Lambda^{\frac{1}{N+1}}\left(H_{2} W u, W u\right)_{L^{2}\left(\mathbb{R}^{2 n+1}\right)} & =\Lambda^{\frac{1}{N+1}} \int_{\mathbb{R}^{2 n+1}} H_{2}(t, X, \Lambda)|\Phi(t, X)|^{2} d t d X,
\end{aligned}
$$

if $\Phi(t, X)=W(u(t, \cdot))(X)$. We can now use the following $L^{2}$-norm splitting

$$
\begin{aligned}
\Lambda^{\frac{1}{N+1}} \int_{\mathbb{R}^{2 n+1}} H_{2}|\Phi|^{2} d t d X= & \Lambda^{\frac{1}{N+1}} \int_{\left\{|Q|<\Lambda^{1 /(N+1)}\right\}} H_{2}|\Phi|^{2} d t d X \\
& +\Lambda^{\frac{1}{N+1}} \int_{\left\{|Q| \geq \Lambda^{1 /(N+1)}\right\}} H_{2}|\Phi|^{2} d t d X \\
\leq & \Lambda^{\frac{1}{N+1}} \int_{\left\{|Q|<\Lambda^{1 /(N+1)}\right.} H_{2}|\Phi|^{2} d t d X \\
& +\int_{\mathbb{R}^{2 n+1}} H_{2}|Q||\Phi|^{2} d t d X,
\end{aligned}
$$

to deduce from Lemma 4.2.2, Lemma 4.2.6 and (219) that there exists a positive constant $c_{21}$ such that for all $u \in C_{0}^{\infty}\left(\mathbb{R}^{n+1}\right)$ and $\Lambda \geq \Lambda_{0}$,

$$
\begin{aligned}
\Lambda^{\frac{1}{N+1}}\left(H_{2}^{\mathrm{Wick}} u, u\right)_{L^{2}} \leq c_{21}\left\|D_{t} u+i Q^{\mathrm{Wick}} u\right\|_{L^{2}}\|u\|_{L^{2}} & +c_{21}\|u\|_{L^{2}}^{2} \\
& +\frac{1}{2} \Lambda^{\frac{1}{N+1}}\|u\|_{L^{2}}^{2} .
\end{aligned}
$$

It follows from (218) and (220) that for all $u \in C_{0}^{\infty}\left(\mathbb{R}^{n+1}\right)$ and $\Lambda \geq \Lambda_{0}$,

$$
\begin{aligned}
& \frac{1}{2} \Lambda^{\frac{1}{N+1}}\|u\|_{L^{2}}^{2} \leq c_{1}^{-1} \Lambda^{-\frac{N}{N+1}}\left\|D_{t} u+i Q^{\mathrm{Wick}} u\right\|_{L^{2}}^{2} \\
& \quad+c_{21}\left\|D_{t} u+i Q^{\mathrm{Wick}} u\right\|_{L^{2}}\|u\|_{L^{2}}+\left(c_{1}^{-1} \Lambda^{-\frac{N}{N+1}}+c_{21}\right)\|u\|_{L^{2}}^{2}
\end{aligned}
$$

Since from (6), $N \in \mathbb{N}^{*}$, we deduce from (221) that there exist some positive constants $c_{22}$ and $\Lambda_{1} \geq 1$ such that for all $u \in C_{0}^{\infty}\left(\mathbb{R}^{n+1}\right)$ and $\Lambda \geq \Lambda_{1}$,

$$
\begin{aligned}
& c_{22} \Lambda^{\frac{1}{N+1}}\|u\|_{L^{2}}^{2} \leq \Lambda^{-\frac{N}{N+1}}\left\|D_{t} u+i Q^{\mathrm{Wick}} u\right\|_{L^{2}}^{2}+\left\|D_{t} u+i Q^{\mathrm{Wick}} u\right\|_{L^{2}}\|u\|_{L^{2}}, \\
& \text { томе } 136 \text { - } 2008-\mathrm{N}^{\mathrm{O}} 3
\end{aligned}
$$


i.e.,

$$
\begin{aligned}
c_{22} \Lambda^{\frac{2}{N+1}}\|u\|_{L^{2}}^{2} \leq \Lambda^{\frac{1-N}{N+1}}\left\|D_{t} u+i Q^{\mathrm{Wick}} u\right\|_{L^{2}}^{2} & \\
& +\Lambda^{\frac{1}{N+1}}\left\|D_{t} u+i Q^{\mathrm{Wick}} u\right\|_{L^{2}}\|u\|_{L^{2}}
\end{aligned}
$$

Since we have

$$
\Lambda^{\frac{1}{N+1}}\left\|D_{t} u+i Q^{\mathrm{Wick}} u\right\|_{L^{2}}\|u\|_{L^{2}} \leq \frac{c_{22}}{2} \Lambda^{\frac{2}{N+1}}\|u\|_{L^{2}}^{2}+\frac{1}{2 c_{22}}\left\|D_{t} u+i Q^{\mathrm{Wick}} u\right\|_{L^{2}}^{2}
$$

we deduce from (222) that there exists a positive constant $c_{23}$ such that for all $u \in C_{0}^{\infty}\left(\mathbb{R}^{n+1}\right)$ and $\Lambda \geq \Lambda_{1}$,

$$
\left\|D_{t} u+i Q^{\mathrm{Wick}} u\right\|_{L^{2}}^{2} \geq c_{23} \Lambda^{\frac{2}{N+1}}\|u\|_{L^{2}}^{2},
$$

which in view of (45) proves the estimate (44) and ends the proof of the theorem 2.1.1.

\section{BIBLIOGRAPHY}

[1] J.-M. Bony - "Sur l'inégalité de Fefferman-Phong", in Seminaire: Équations aux Dérivées Partielles, 1998-1999, Sémin. Équ. Dériv. Partielles, exposé $\mathrm{n}^{\circ}$ III, École Polytech., 1999, p. 16.

[2] A. Boulkhemair - " $L^{2}$ estimates for Weyl quantization", J. Funct. Anal. 165 (1999), p. 173-204.

[3] E. B. DAvies - "Pseudo-spectra, the harmonic oscillator and complex resonances", R. Soc. Lond. Proc. Ser. A Math. Phys. Eng. Sci. 455 (1999), p. $585-599$.

[4] _ "Semi-classical states for non-self-adjoint Schrödinger operators", Comm. Math. Phys. 200 (1999), p. 35-41.

[5] N. Dencker, J. Sjöstrand \& M. Zworski - "Pseudospectra of semiclassical (pseudo-) differential operators", Comm. Pure Appl. Math. 57 (2004), p. 384-415.

[6] L. HÖRMANDER - "A class of hypoelliptic pseudodifferential operators with double characteristics", Math. Ann. 217 (1975), p. 165-188.

[7] _ The analysis of linear partial differential operators, Springer, 1983-1985.

[8] N. LERNER - "Energy methods via coherent states and advanced pseudodifferential calculus", in Multidimensional complex analysis and partial differential equations (São Carlos, 1995), Contemp. Math., vol. 205, Amer. Math. Soc., 1997, p. 177-201.

[9] _ "The Wick calculus of pseudo-differential operators and some of its applications", Cubo Mat. Educ. 5 (2003), p. 213-236. 
[10] K. Pravda-Starov - "A complete study of the pseudo-spectrum for the rotated harmonic oscillator", J. London Math. Soc. (2) 73 (2006), p. 745761.

[11] L. N. TRefethen - "Pseudospectra of linear operators", SIAM Rev. 39 (1997), p. 383-406.

[12] M. ZWORSKI - "Numerical linear algebra and solvability of partial differential equations", Comm. Math. Phys. 229 (2002), p. 293-307. 